\title{
Amplitude Death: \\ The emergence of stationarity in coupled nonlinear systems
}

\author{
Garima Saxena $^{\mathrm{a}}$, Awadhesh Prasad*a,b, Ram Ramaswamy ${ }^{\mathrm{c}, \mathrm{d}}$ \\ ${ }^{a}$ Department of Physics and Astrophysics, University of Delhi, Delhi 110007, India \\ ${ }^{b}$ MPI-PKS, Nöthnitzer Strasse 38, 01187 Dresden, Germany \\ ${ }^{c}$ School of Physical Sciences, Jawaharlal Nehru University, New Delhi 110067, India \\ ${ }^{d}$ University of Hyderabad, Hyderabad 500046, India
}

\begin{abstract}
When nonlinear dynamical systems are coupled, depending on the intrinsic dynamics and the manner in which the coupling is organized, a host of novel phenomena can arise. In this context, an important emergent phenomenon is the complete suppression of oscillations, formally termed amplitude death (AD). Oscillations of the entire system cease as a consequence of the interaction, leading to stationary behavior. The fixed points that the coupling stabilizes can be the otherwise unstable fixed points of the uncoupled system or can correspond to novel stationary points. Such behaviour is of relevance in areas ranging from laser physics to the dynamics of biological systems. In this review we discuss the characteristics of the different coupling strategies and scenarios that lead to $\mathrm{AD}$ in a variety of different situations, and draw attention to several open issues and challenging problems for further study.
\end{abstract}

Keywords: Amplitude Quenching, Interaction, Synchronization, Fixed-point solution, Control, Network, Bifurcation

Email addresses: gsaxena1@physics.du.ac.in (Garima Saxena), awadhesh@physics.du.ac.in (Awadhesh Prasad*), rr@uohyd.ernet.in (Ram Ramaswamy)

${ }^{*}$ Corresponding Author: Fax +11-2766 7061; Phone +11-2766 7725/Extn. 1354. 


\section{Contents}

1 Introduction

2 Scenarios for Amplitude Death 6

2.1 Mismatched oscillators . . . . . . . . . . . . . . . 7

2.2 Delay interaction . . . . . . . . . . . . . . . . 11

2.3 Conjugate coupling . . . . . . . . . . . . . . . 13

2.4 Dynamic coupling . . . . . . . . . . . . . . . . 14

2.5 Nonlinear Coupling . . . . . . . . . . . . . . . . 15

2.6 Linear augmentation, and other strategies . . . . . . . . . 16

2.7 Velocity Coupling . . . . . . . . . . . . . . . . 17

2.8 Routes to AD . . . . . . . . . . . . . . . . . . . . . . . . . . . . . . . . .

2.8.1 Hopf bifurcation ............... . . . 20

2.8.2 Saddle-node Bifurcation . . . . . . . . . . . 20

2.8.3 Direct transition ............... . . 21

3 Targeting and Control 23

3.1 Steady-states through nonlinear coupling . . . . . . . . . . 23

3.1.1 Linear Augmentation . . . . . . . . . . . . . . . . 27

3.2 Reprieve: Avoiding fixed points . . . . . . . . . . . . . . . 28

3.2.1 Gradient coupling . . . . . . . . . . . . . . . . 29

4 Characterization 30

4.1 The phase-flip ....................... 32

5 Amplitude death on networks 35

6 Experiments and applications 39

7 Summary

8 Acknowledgments 43

9 References 43 


\section{Introduction}

The nature of the dynamics in coupled nonlinear systems is a central issue of interest in a number of areas of scientific enquiry. A variety of natural phenomena-ranging from the motion of pendulums or springs, to lasers, fluid flow, ecological systems, populations, and so on-are modeled as oscillators. These may be linear or nonlinear, depending on the context, and as is well known, the motion can be periodic or chaotic. When systems that are in isolation capable of complex behaviour are coupled, a host of novel phenomena can be seen. These depend both upon the properties of the isolated systems - the nature of the nonlinearity, whether the motion is chaotic or regular, what are the equilibria, and so on-as well as the manner in which their coupling is organized.

An important emergent phenomenon in this context is the suppression of oscillations, formally termed amplitude death (AD): as a consequence of the interaction oscillations of the entire system cease, leading to stationarity [1, 2, 3]. Such behaviour is emergent in the sense that the isolated or uncoupled systems do not exhibit stationary dynamics. In a formal sense, amplitude death is an instance of a more general phenomenon that includes both the cessation of oscillations as a consequence of coupling, as well the suppression of amplitude variations, namely the conversion of chaotic oscillations to periodic or quasiperiodic dynamics. In a sense, this is the stabilization or creation of "simpler" attractors through the coupling.

When oscillations stop, there are two possibilities for AD. If the coupled systems have exactly one equilibrium, then the occurrence of amplitude death implies that this equilibrium becomes asymptotically stable. However, the coupled systems can have more than one stationary state and then one can have the stabilization of new fixed points, namely those that are not stable (or may not even exist) in the uncoupled system.

A general setting in which we discuss AD in this review is a configuration of $N$ coupled nonlinear oscillators that is specified by the equation

$$
\dot{\mathbf{X}}_{i}=\mathbf{F}_{i}\left(\mathbf{X}_{i}\right)+\varepsilon \mathbf{G}_{i}(\mathbf{X}) \quad i=1, \ldots, N
$$

where

$$
\mathbf{G}_{i}(\mathbf{X})=\frac{1}{K_{i}} \sum_{j=1}^{N} A_{i j} \mathbf{H}\left(\mathbf{X}_{i}, \mathbf{X}_{j}, \tau\right), \quad i=1, \ldots, N
$$


Here $\mathbf{X}_{i}$ is the $m_{i}$-dimensional vector of dynamical variables for the $i$ th oscillator, and the $\mathbf{F}_{i}$ 's specify their evolution equations. The oscillators may be identical or distinct, and are coupled to other oscillators, as specified via the function $\mathbf{G}_{i}$. $K_{i}$ is a normalization factor, $\varepsilon$ is the coupling strength, and the connection topology is specified by the connectivity matrix, whose elements $A_{i j}$ are 1 or 0 , depending on whether oscillators $i$ and $j$ are coupled with each other or not. The actual coupling is specified by the term $\mathbf{H}\left(\mathbf{X}_{i}, \mathbf{X}_{j}, \tau\right)$ which is a function of $\mathbf{X}_{i}(t)$ and $\mathbf{X}_{j}(t-\tau)$. The time-delay $\tau$ can be fixed, or can have a more complex dependence on time. Most of the major results discussed in this review are illustrated with two coupled oscillators, $N=2$.

Shown in Fig. 1 is a schematic of the basic phenomenology. Consider two oscillators coupled by some function $\mathbf{G}$. When the coupling strength $\varepsilon=0$ the motion of individual subsystems are oscillations (whether chaotic (C), periodic $(\mathrm{P})$ or quasiperiodic $(\mathrm{QP})$ ); a simple periodic case is illustrated in Fig. 1(a). When the coupling is switched on, above a specific strength when amplitude death occurs, the transient dynamics is essentially that of damped oscillations, as shown in Figs. 1 (b) and (c). As can be seen, although this is an amplitude phenomenon, there could be additional effects that involve the phases of the oscillators, and indeed, any analytic understanding of this phenomenon must include a discussion of both the amplitudes and the phases of the interacting systems.

There are some terminological issues that need to be clarified. In this article we will term the cessation of oscillations, regardless of what fixed points are stabilized asymptotically, as amplitude death, even though the asymptotic steady states in the different subsystems may not be the same (as for example in Fig. 1(c)) and can be different from the null state, $\mathbf{X}_{i}^{*} \equiv 0$. This heterogeneous case has been termed "oscillation death" [1, 4, 5] or the BarEli Effect [6]), keeping the term amplitude death for the homogeneous case, when a simple transformation can shift the fixed point to the origin [6, 7] (as for example in Fig. 1(b)). What these fixed points are will naturally depend on the internal structure of the individual oscillators, whether they are identical or mismatched, whether the coupling is symmetric or asymmetric, and indeed what the nature of the coupling is, namely whether the interactions are diffusive or nonlinear.

Following the initial work by Bar-Eli [1] AD has been studied quite extensively [6, 8, 9, 10]. Different situations where amplitude death occurs have been described, and it is now known that the phenomenon can be seen when the interacting systems are identical [7, 11, 12, 13], mismatched [1, 6], when 
a)
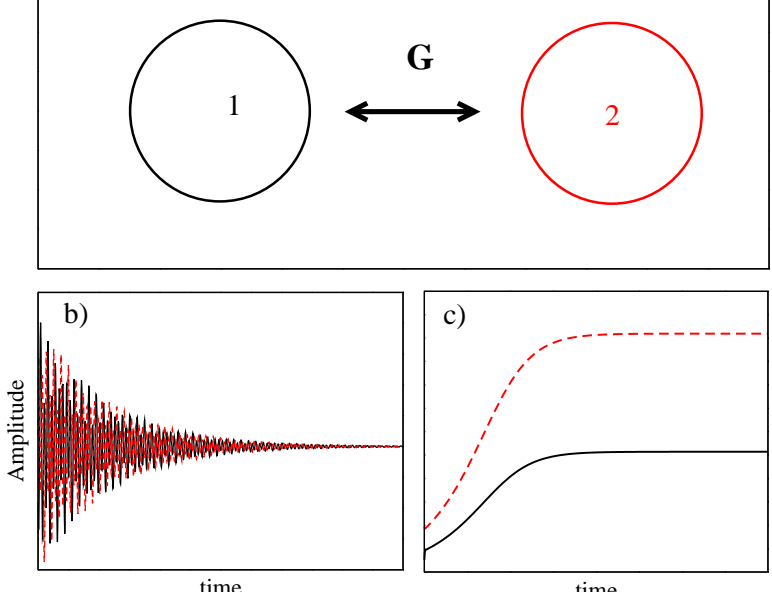

Figure 1: (Color online) Schematic of the dynamics for a typical case of two oscillators with a given coupling function $\mathbf{G}$, showing (a) the phase space dynamics of the uncoupled oscillators, namely when $\varepsilon=0$, and the transient motions of the two subsystems (solid and dashed lines for respective oscillators) as a function of time, leading to either (b) the same (homogeneous) or (c) the distinct (heterogenous) steady states respectively for appropriate coupling strength, $\varepsilon \neq 0$. 
the coupling is dynamical [14], or nonlinear (nondiffusive) [15]. We review these various situations and also discuss the general mechanism for AD in Sec. 2,

In many practical situations $\mathrm{AD}$, is desirable, as for example in laser applications [16, 17, 18, 19, 20, 21] where a constant output is needed and fluctuations should be suppressed. There are also other situations where oscillations need to be maintained, as for instance in brain functioning [22, 23. These different requirements suggest that control strategies to either to achieve or to avoid AD in coupled systems are necessary, and in Sec. 3 we review some control methods that have been employed to either target or avoid steady states in coupled systems.

As indicated above, understanding the transient dynamics is important in describing AD since this state is asymptotically featureless. Transient dynamics can be significant in many applications that are restricted to finite times, as for example in ecology [24]. Furthermore, since the different subsystems are coupled, a study of the synchronization properties [25] is also of interest. In a number of examples it has been noted that the individual systems, while being synchronized, can nevertheless be either in-phase or out of phase, with a transition between these states at a specific value of the coupling [26, 27, 28]. We review this phase-flip transition and its associated behaviour in Sec. 4 .

The occurrence of AD in networks of coupled oscillators [15, 29, 30, 31, 32, 33, 34, 35] is another topic of considerable current relevance. Studies have been carried out for a variety of network topologies, ranging from systems coupled on a ring [36, 37], as well as in the so-called small world [38] and scale-free networks [39]. These are discussed in Sec. 5],

Our principal aim in this review on amplitude death is to stress the importance of this phenomenon and its applications in real situations. The major experimental results and possible applications are discussed in Sec. 6. Finally, the review concludes in Sec. 7 with a summary.

\section{Scenarios for Amplitude Death}

To begin with, we consider the several known scenarios within which AD is known to occur, as well as constraints and necessary conditions. One aspect of interest is in identifying the different types of interactions that facilitate $\mathrm{AD}$. This is of particular relevance in natural systems where the parameter of individual systems may not be accessible, and amplitude death can be 
achieved mainly by selecting appropriate forms of the interaction. It is also possible that for a given form of the interaction, AD occurs when internal parameters are varied. As a parallel, consider a related phenomenon, the synchronization of two systems. There are specific forms of interaction that cause systems to synchronize, and for a specific form of coupling - diffusive, say - synchrony is possible only when the coupling strength is sufficiently strong.

The Landau-Stuart limit cycle oscillator system [6, 40, 41] has been an ideal model for the exploration of several of the scenarios we discuss here. This is a two-dimensional oscillator given by the evolution equation

$$
\dot{Z}=\left[1+i \omega-|Z|^{2}\right] Z,
$$

where the complex variable $Z=x+i y$. As can be seen, the origin is an unstable fixed point, and the unit circle is an attractor for positive frequency, $\omega$. The relative simplicity of the evolution equations makes the analysis tractable, including the case of time-delayed interactions.

In this section the case of two oscillators is considered, and some numerical results are presented first. The stability of the fixed points can be estimated by computing the eigenvalues of the Jacobian matrix. When all eigenvalues have negative real parts, the entire spectrum of Lyapunov exponents [42] is negative: this is the state of $\mathrm{AD}$. In the numerical results presented here the standard Runge-Kutta scheme [43] was used.

\subsection{Mismatched oscillators}

An early observation of amplitude quenching was reported by Crowley and Field [44] who experimentally coupled two chemical oscillators, the Belousov-Zhabotinski system electrically. The coupling essentially involved the mass transfer of a single species, the $\mathrm{Ce}^{3+}$ ions, and caused the composite system to reach a state wherein one of the systems was quenched to a steady state, while the other maintained oscillations. This experiment served as the starting point for the study of this behaviour by Bar-Eli [1] who showed that two interacting model continuous stirred tank reactors can stop oscillations and arrive at steady states if coupled diffusively. Consider the cell Brusselator model [1], defined as

$$
\begin{aligned}
& \dot{x}=-(B+1) x+x^{2} y+A \\
& \dot{y}=B x-x^{2} y
\end{aligned}
$$


where $x$ and $y$ are the dimensionless concentrations of two chemical species [3, 1] while the (positive) parameters $A$ and $B$ are the rate constants for the production of $x$ and $y$. This system has one steady state, $x^{*}=A, y^{*}=B / A$ which is unstable if $A^{2}<(B-1)$. The oscillating behavior of this system is shown in Fig. 2(a) for parameters $A=5$ and $B=35$. When two such cells are coupled diffusively [1] then the dynamical equations take the form (the variables of the two systems are distinguished by subscripts)

$$
\begin{aligned}
& \dot{x_{1}}=-\left(B_{1}+1\right) x_{1}+x_{1}^{2} y_{1}+A_{1}+\varepsilon\left(x_{2}-x_{1}\right) \\
& \dot{y_{1}}=B_{1} x_{1}-x_{1}^{2} y_{1}+\varepsilon\left(y_{2}-y_{1}\right)
\end{aligned}
$$

with corresponding equations for subsystem 2 . The coupling terms $\varepsilon\left(x_{2}-x_{1}\right)$ and $\varepsilon\left(y_{2}-y_{1}\right)$ arise from material transfer between the cells through diffusion, and the coupling strength $\varepsilon$ corresponds to the rate of this transfer. The largest two Lyapunov exponents at $A_{1}=A_{2}=5, B_{1}=B_{2}=35$ as a function of the coupling strength in Fig. 2(b). The transition to AD can be identified as the point when all Lyapunov exponents become nonpositive (and which then correspond to the real parts of the eigenvalues of the fixed point-see Sec. (4). Both the oscillators then settle to different steady states.

Transient trajectories for both oscillators are shown in Figs. 2(c) and (d), demonstrating that this is a case of oscillation death: the two fixed points $\left(x_{1}^{*}=0.34, y_{1}^{*}=22.36\right)$ and $\left(x_{2}^{*}=9.66, y_{2}^{*}=3.72\right)$ are distinct, and different from the fixed point of the uncoupled systems, namely $x^{*}=5, y^{*}=7$.

Similar behavior was found to exist in other well-known chemical oscillators [1, 45], including the Noyes-Field-Thompson model, the Oregonator, and the Field-körös-Noyes models for the Belousov-Zhabotinski reaction, the model of first-order decomposition autocatalysis [46] and the LotkaVolterra model for prey-predator interactions. These systems differ significantly showing that the occurrence of amplitude death is fairly general. This extensive numerical analysis [1] triggered a serious exploration of the phenomenon of amplitude death from both a theoretical and experimental point of view.

Analysis by Aronson, Ermentrout, and Kopell [6] provided a deeper mathematical understanding of this phenomenon. They considered the following system of two Landau-Stuart oscillators,

$$
\begin{aligned}
& \dot{Z}_{1}=\left[1+i \omega_{1}-\left|Z_{1}\right|^{2}\right] Z_{1}+\varepsilon\left[Z_{2}-Z_{1}\right], \\
& \dot{Z}_{2}=\left[1+i \omega_{2}-\left|Z_{2}\right|^{2}\right] Z_{2}+\varepsilon\left[Z_{1}-Z_{2}\right]
\end{aligned}
$$



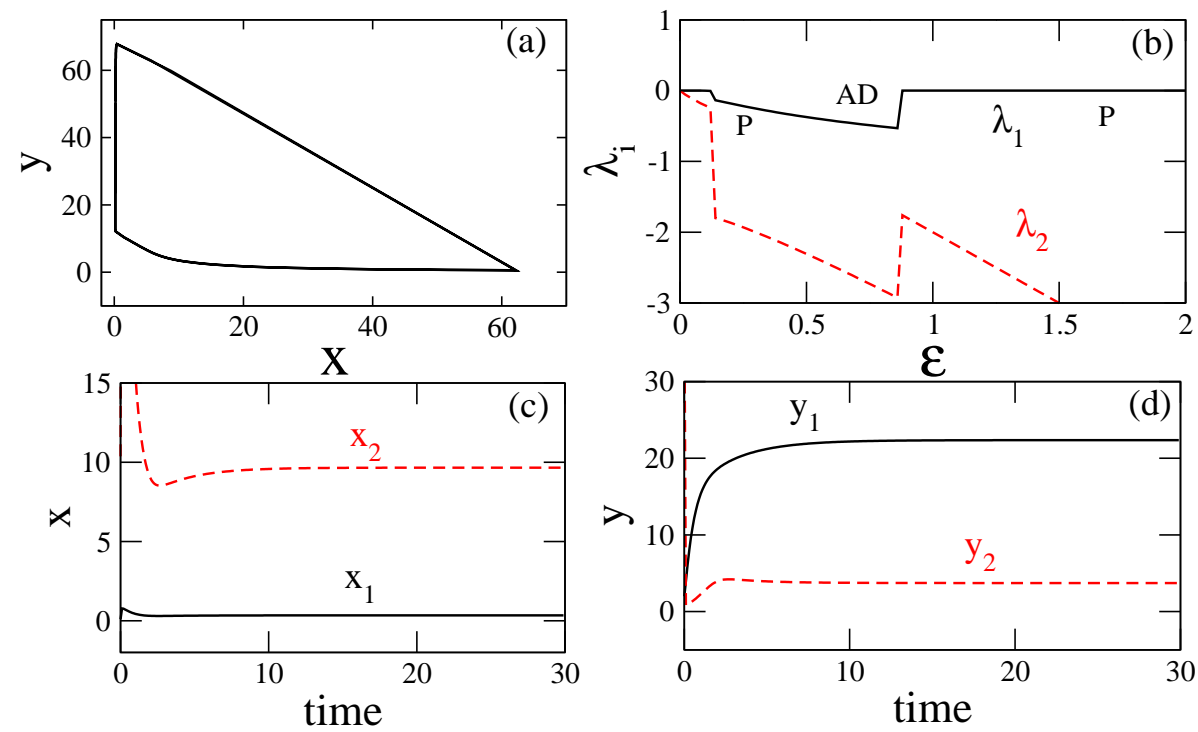

Figure 2: (Color online) (a) The trajectory of a single Brusselator, Eq. (4), at parameters $A=5$ and $B=35$ in phase space. (b) The largest two Lyapunov exponents as a function of coupling strength $\varepsilon$ for two coupled identical Brusselators, Eq. (5) at parameters $A_{1}=$ $A_{2}=5$ and $B_{1}=B_{2}=35$. (c) And (d) show the transient trajectories of both oscillators as function of time at coupling strength $\varepsilon=0.5$. 


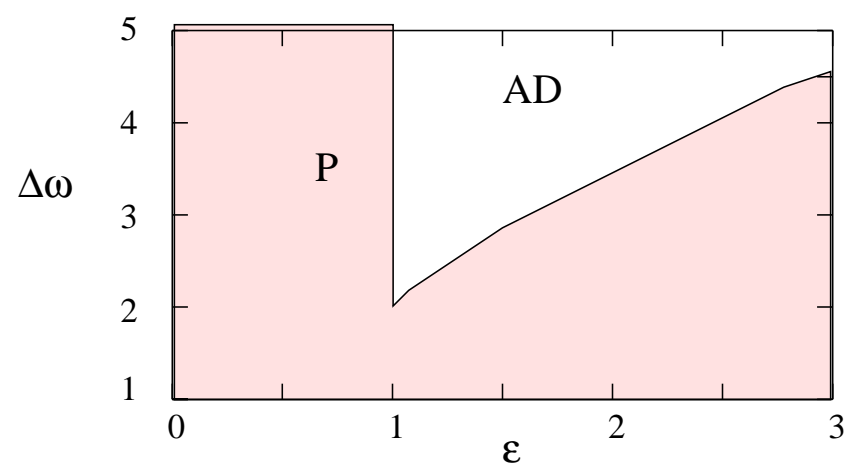

Figure 3: The phase diagram in the parameters: frequency mismatch $(\Delta \omega)$ and coupling strength $(\varepsilon)$ of Eq. (6). The shaded and unshaded regions correspond to the periodic $(\mathrm{P})$ and amplitude death (AD) motions respectively. For detail see Ref. [6].

where the notation is obvious. When uncoupled, both oscillators have an unstable fixed point at $Z_{1,2}^{*}=0$, and the unit circles $\left|Z_{1,2}\right|=1$ are limit cycles. Depending on the mismatch $\Delta \omega=\left|\omega_{1}-\omega_{2}\right|$, this system shows different types of dynamics [6]. Shown in Fig. [3 is a schematic phasediagram, indicating the different dynamical states: the shaded and blank regions correspond to periodic motion and $\mathrm{AD}$ respectively. For a specific value of the mismatch, $\Delta \omega=4$, the largest two Lyapunov exponents are shown in Fig. 4, as a function of the coupling. The transient dynamics in the $\mathrm{AD}$ region is shown in the inset; note that this is a case of homogenous steady states and both oscillators settle onto the (now stabilized) fixed point, the origin $Z_{i}^{*}=0$. Linear stability analysis in the neighborhood of the origin shows that amplitude death occurs for $\varepsilon>1$ and $\Delta \omega>2 \sqrt{2 \varepsilon-1}$.

The sign of $\omega$ determines the sense of rotation in phase. In Eq. (6) the frequencies are both positive so that in the phase plane the direction of motion for both oscillators is the same. In this case, the systems are termed corotating, while when the frequencies differ in sign, they are counter-rotating 477. This latter situation is one of parameter mismatch, and in recent works [47, 48, 49], the characteristics of $\mathrm{AD}$ in such cases have been discussed; see also Sec. 4 .

Parameter mismatch is thus one of the major causes of amplitude death, and has been extensively studied both analytically and experimentally [4, 5, 6, 9, 32, 47]. 


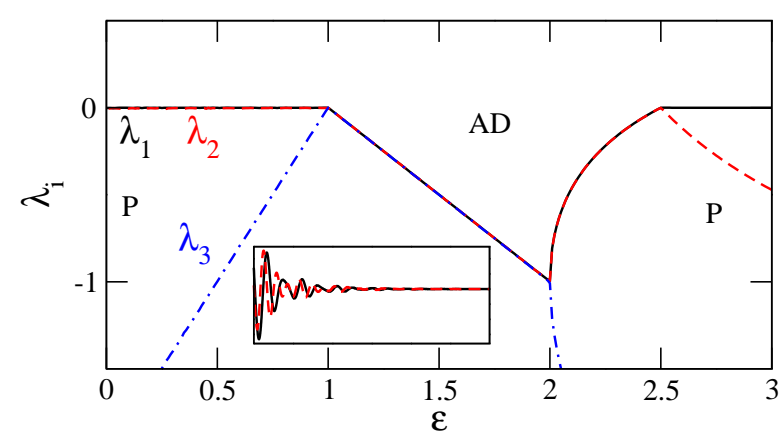

Figure 4: (Color online) The largest two Lyapunov exponents as a function of coupling strength, at $\Delta \omega=4\left(\omega_{1}=4\right.$ and $\left.\omega_{2}=8\right)$, Eq. (6). Inset figure shows in-phase synchronous behaviour of transient trajectories of both the oscillators as a function of time at coupling strength $\varepsilon=1.5, x_{1}$ (solid-black line) and $x_{2}$ (dashed-red line).

\subsection{Delay interaction}

In the above systems, the interaction is considered to be instantaneous, namely the coupling terms involve the variables of the two subsystems at the same time, $t$. In many physical systems, the coupling involves time-delay, and this was considered by Reddy, Sen and Johnston [7] who investigated the collective dynamical behavior of limit-cycle oscillators interacting diffusively via time-delayed coupling. Here AD was achieved even in the absence of mismatch, and was demonstrated in an experiment involving electronic circuits. As was shown through both analysis and numerics [7, 50, 51, 52, 53], there is a significant region in the parameter space of coupling strength and time delay where AD occurs.

The delay-coupled Landau-Stuart system has the evolution equations

$$
\begin{aligned}
& \dot{Z}_{1}=\left[1+i \omega_{1}-\left|Z_{1}\right|^{2}\right] Z_{1}+\varepsilon\left[Z_{2}(t-\tau)-Z_{1}\right], \\
& \dot{Z}_{2}=\left[1+i \omega_{2}-\left|Z_{2}\right|^{2}\right] Z_{2}+\varepsilon\left[Z_{1}(t-\tau)-Z_{2}\right],
\end{aligned}
$$

where the output of one system takes a time $\tau$ to influence the other, as might occur in spatially separated systems when there is a finite speed of signal transmission.

Consider identical systems, $\omega_{1}=\omega_{2}=6$. Shown in Fig. 5 is the largest two Lyapunov exponents as a function now of the time-delay $\tau$ for fixed coupling strength $\varepsilon=2$. This clearly shows the regime of AD when all Lyapunov exponents are negative (see details in Sec. 2.8). Transient trajectories (shown in the inset) for both oscillators indicates the cessation of oscillations 


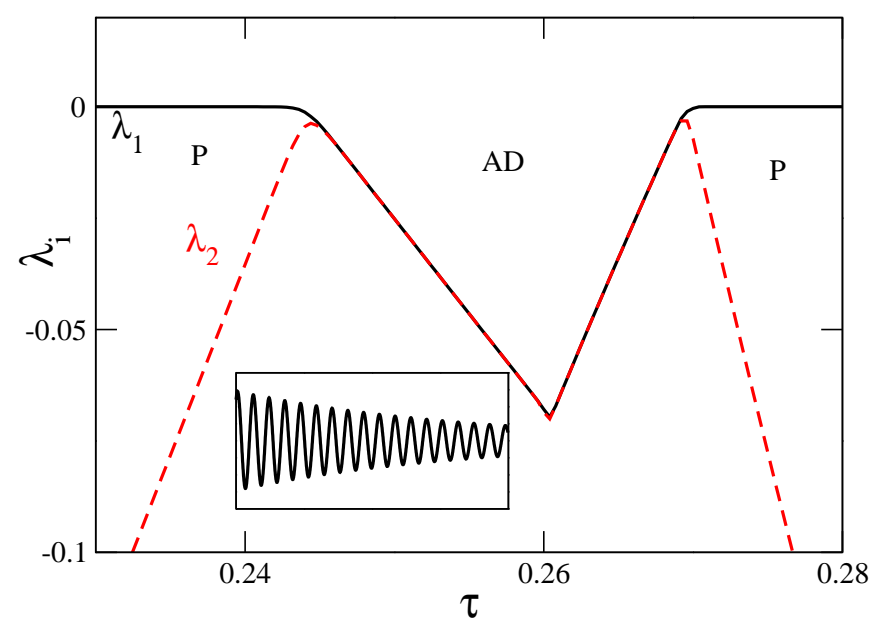

Figure 5: (Color online) The largest two Lyapunov exponents as a function of coupling strength for coupled identical oscillators for $\Delta \omega=0\left(\omega_{1}=6\right.$ and $\left.\omega_{2}=6\right)$, (Eq. (7)). Inset figure: completely synchronized transient trajectories, $x_{1}$ (solid-black line) and $x_{2}$ (dashed-red line), as a function of time at time-delay $\tau=0.255[$ [, 26] .

and the stabilization of the homogeneous steady state, $Z_{1,2}^{*}=0$. The $\mathrm{AD}$ in such delayed coupled systems has a resonance-like character, occurring in repeated "islands" in the parameter space of $\varepsilon$ and $\tau$ [7, 50, 51, 52, 53].

This scenario for the occurrence of $\mathrm{AD}$ in delay-coupled systems is quite general and does not depend on the nature of the dynamics in the uncoupled systems. As was shown by Prasad [26], even chaotic oscillators can be stabilized in this manner. The importance of time delay interactions has been highlighted in [54].

Given a finite transmission speed for signals from one subsystem to reach the other, a natural extension is to consider the situation when the timedelay itself varies. This is particularly important when there are stochastic effects that are being modeled by the delay itself. Atay [13] considered the effect of a distribution of time-delays and showed that even a small spread in the delay distribution can greatly enlarge the set of parameters for which amplitude death occurs.

The expansion of the AD region with a distribution of delays is a important effect with a wide range of applications [13], particularly since if the variance of the distribution is above a threshold, this $\mathrm{AD}$ region can become unbounded. Again, the effect is quite general, occurring for arbitrary spread in the delay, for different forms of the distribution, and for arbitrary numbers 
of oscillators [13, 55]. Closely related to the case of distributed delays is the situation where systems respond to cumulative signals, namely by integrating information received over an interval in time. This occurs when systems have a finite intrinsic response time and also causes the region of AD to extend indefinitely [56, 57].

Since time-delay interactions are common in experiments, various aspects of both fixed and distributed delays, including a deterministic time-varying interaction [58, 59] have been studied. One application is to the stabilization of fixed points [60, 61, 62]. Velocity delayed coupling has been also explored in the context of amplitude death [63, 64] (see Sec. 2.7). Mixed interaction schema, with instantaneous interactions in one system and time-delay in the other [65] or unequal time-delays in different directions (to model spatial heterogeneity [66, 67] and one-way ring time-delay [37] have been studied in recent works.

\subsection{Conjugate coupling}

A novel context for amplitude death is when systems are coupled via so-called conjugate or dissimilar variables. With such coupling, the necessity for systems being either mismatched or having time-delayed interactions can be dropped, and AD occurs in identical systems with instantaneous coupling. This was first studied in a model system by Karnatak et al. [11] who considered two Landau-Stuart oscillators with the evolution equations

$$
\begin{aligned}
& \dot{x}_{1}=P_{1} x_{1}-\omega_{1} y_{1}+\varepsilon\left(y_{2}-x_{1}\right) \\
& \dot{y}_{1}=P_{1} y_{1}+\omega_{1} x_{1}+\varepsilon\left(x_{2}-y_{1}\right)
\end{aligned}
$$

where $P_{i}=1-\left|Z_{i}\right|^{2}$, and similar equations can be written for subsystem 2 . Note that here the $y$ variable of the second oscillator is diffusively coupled to the $x$ variable of the first oscillator and vice versa.

Coupling via conjugate variables is natural in a variety of experimental situations, and indeed the above system was inspired by the experiments of Kim and Roy [16, 17] on coupled semiconductor laser systems where the photon intensity fluctuations from one laser were used to modulate the injection current of a second identical laser and vice versa. Indeed it would not be possible to couple two lasers through their intensity fluctuations directly. As it happens, in a model of the semiconductor laser, the current and the fluctuations are, in a sense, conjugate variables that have constitute the chaotic oscillator system [16, 17]. The largest three Lyapunov exponents for 


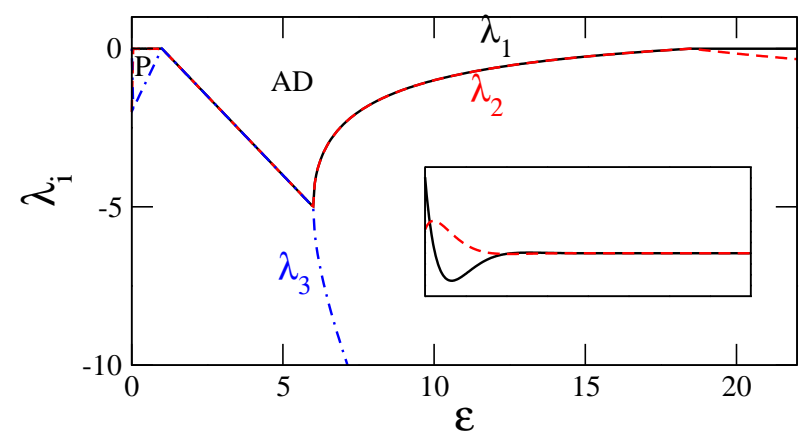

Figure 6: (Color online) The largest three Lyapunov exponents as a function of coupling strength at $\Delta \omega=0\left(\omega_{1}=6\right.$ and $\left.\omega_{2}=6\right)$, Eq. (8). Inset figure: the transient trajectories, $x_{1}$ (solid-black line) and $x_{2}$ (dashed-red line), as a function of time at coupling strength $\varepsilon=5[11]$.

the above system is shown in Fig. 6, and as can be seen, there is a wide range of coupling strength where all Lyapunov exponents are negative, indicating stabilization of the fixed point. A transient trajectories are shown in the inset.

A detailed analytical condition for the occurrence of amplitude death can be found in [68]. With conjugate coupling, AD can be anticipated by recognizing that there is a strong analogy between time-delayed and conjugate variables: as a matter of fact, the Takens' embedding theorem [69] asserts that the topological properties of a dynamical system that is reconstructed through delay variables match those of the true system for appropriate choices of embedding dimension and time delay. Thus using conjugate variables is akin to using time-delay coupling, and this gives rise to regimes of $\mathrm{AD}$.

In addition, there are other new features such as the suppression of chaos, and riddling [11, 12, 70] due to conjugate coupling. In addition, this is particularly suited to experiments and both in electrochemical systems [71] as well as in electronic circuits [72], this coupling can be realized.

\subsection{Dynamic coupling}

Konishi [14] has proposed another type of interaction which, in contrast to the cases discussed above has an evolving or "dynamic" coupling. Consider the Landau-Stuart system

$$
\dot{x}_{1}=P_{1} x_{1}-\omega_{1} y_{1}+\varepsilon\left(u_{1}-x_{1}\right)
$$




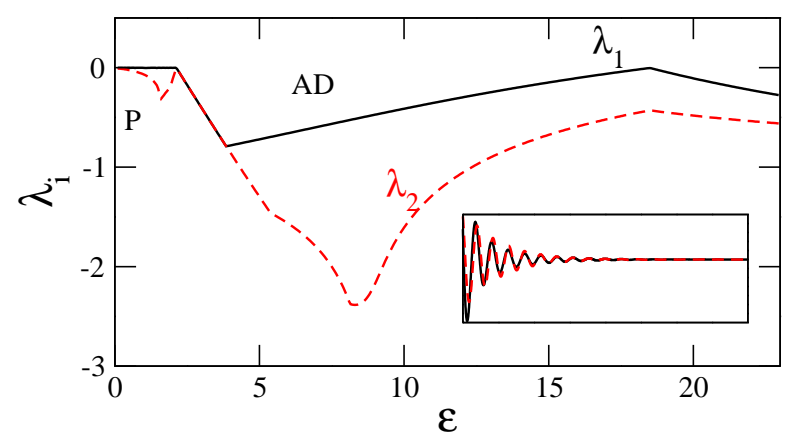

Figure 7: (Color online) The largest two Lyapunov exponents as a function of coupling strength, at $\Delta \omega=0\left(\omega_{1}=6\right.$ and $\left.\omega_{2}=6\right)$, Eq. (9). Inset figure: transient trajectories, $x_{1}$ (solid-black line) and $x_{2}$ (dashed-red line), as a function of time at coupling strength $\varepsilon=3[14]$.

$$
\begin{aligned}
& \dot{y}_{1}=P_{1} y_{1}+\omega_{1} x_{1} \\
& \dot{u}_{1}=-u_{1}+x_{2}
\end{aligned}
$$

with similar equations for subsystem 2 , and where $P_{i}=1-\left|Z_{i}\right|^{2}$ as usual. The variables $u_{i}$ follow linear dynamics in absence of the $x_{j}$ 's so that the coupling has its own nontrivial dynamics. Shown in Fig. 7 is the largest two Lyapunov exponents; the occurrence of amplitude death is evident, and the transient dynamics are shown in the inset. Note that this instance of amplitude death is in identical systems. In addition to estimating necessary and sufficient conditions for amplitude death to occur, this form of the dynamics has lent itself to extensive analytic study [73, 74, 75].

\subsection{Nonlinear Coupling}

In the preceding examples, the form of the coupling has been taken to be linear or diffusive. An interesting extension that uses nonlinear coupling makes it possible to not only achieve $\mathrm{AD}$, but also to specify the steady state of the coupled system [15, 21]. We will illustrate the targeting of specific fixed points in Sec. 3.1. Consider again the Landau-Stuart oscillators

$$
\begin{aligned}
& \dot{x}_{1}=P_{1} x_{1}-\omega_{1} y_{1}+\varepsilon\left(x_{1}-\alpha\right) \exp \left(x_{2}-\beta\right) \\
& \dot{y}_{1}=P_{1} y_{1}+\omega_{1} x_{1} .
\end{aligned}
$$

where the coupling is nonlinear (and similar equations hold for subsystem 2). The motivation for this choice of function is discussed in Sec. 3.1, and is common in neuronal systems [15, 21]. 


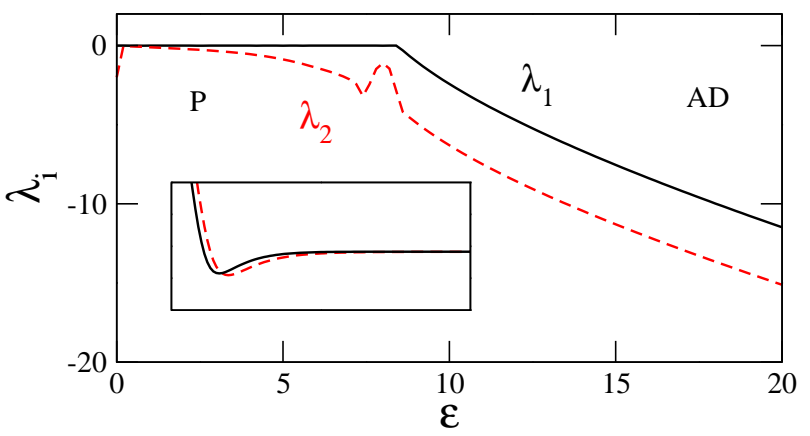

Figure 8: (Color online) The largest two Lyapunov exponents as a function of coupling strength, at $\Delta \omega=0\left(\omega_{1}=6\right.$ and $\left.\omega_{2}=6, \alpha=1\right)$ and $\beta=0.1$, Eq. (10). Inset figure: the transient trajectories, $x_{1}$ (solid-black line) and $x_{2}$ (dashed-red line), as a function of time at coupling strength $\varepsilon=15[15,21$.

Shown in Fig. 8 is the largest two Lyapunov exponents for identical oscillators as a function of $\varepsilon$, the region where all Lyapunov exponents are negative corresponds to amplitude death (the transients are shown in the inset). We have found that the phenomenon of amplitude death when systems are coupled through nonlinear interactions is very general and occurs in the absence of parameter mismatch, in the absence of time-delay (although time delay can enhance the effect) [15, 76].

\subsection{Linear augmentation, and other strategies}

A final scenario we discuss dispenses with the need that the interacting oscillators be similar. Recently Sharma et al. [77] have proposed a new strategy through which a nonlinear oscillator, when coupled to a linear system, experiences amplitude death. Consider such a Landau-Stuart oscillator

$$
\begin{aligned}
& \dot{x}=P x-\omega y+\varepsilon(u-x) \\
& \dot{y}=P y+\omega x \\
& \dot{u}=-k u-\varepsilon(x-\beta) .
\end{aligned}
$$

where $\varepsilon$ is the coupling strength. The variable $u$ describes the dynamics of the linear system with decay parameter $k$ (details are given in Sec. 3.1.1), and the largest two Lyapunov exponents in Fig. 9, clearly shows the region of amplitude death.

Such interaction has been termed linear augmentation, in the sense that the dimension of the oscillator is increased by one through the addition of 


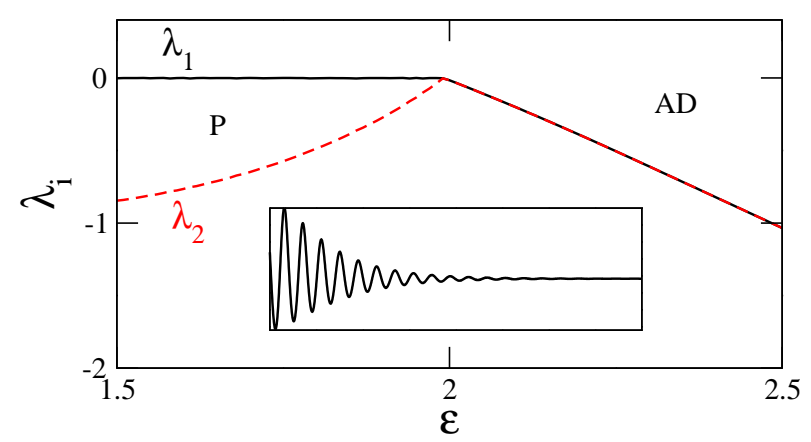

Figure 9: (Color online) The largest two Lyapunov exponents as a function of coupling strength at $\Delta \omega=0\left(\omega_{1}=6\right.$ and $\left.\omega_{2}=6, K=1\right)$ and $\beta=1$, Eq. (11). Inset figure: transient trajectory, $x$, as a function of time at coupling strength $\varepsilon=2$ [77].

the linear equation. This interaction is also dynamical (cf. 2.4), the main difference between the two strategies, Eqs. (9) and (11) is that the interaction of each oscillator is via a linear evolution equation in the former case, while a single oscillator is used as self-feedback in the latter case. Such forms of interaction, Eq. (11), are useful in the context of targeting unstable fixed points, as shown in Sec. 3.1.1.

Apart from above discussed scenarios, other situations where amplitude death occurs include the case of indirect coupling [49, 78, 79]: when two oscillators are coupled via a third, the presence of the intermediate system causes an effective "transmission" delay, which then effects amplitude death. It has been also suggested [78] that the $\mathrm{AD}$ is due to competition between synchronization and anti synchronization. Similarly, the conflict between attractive and repulsive (i.e. negative and positive) diffusive coupling also gives rise to $\mathrm{AD}$ [80]. AD has also been seen in parametrically modulated systems [81], phase repulsive communication [82], and by forcing [83] or gradient coupling [65].

\subsection{Velocity Coupling}

The discussion above has focused entirely on coupled dissipative systems. The Lyapunov spectrum of conservative Hamiltonian systems is constrained by symmetry considerations to have an equal number of positive and negative exponents and to sum to zero. Thus these cannot show AD. However, when two conservative systems are coupled by time-delayed velocity coupling these can show AD 84]. 
Consider, for instance two non-integrable Hénon-Heiles systems [42] with time-delayed velocity coupling as

$$
\begin{aligned}
& \ddot{x}_{1}=-x_{1}-2 x_{1} y_{1}+\varepsilon\left(\dot{x}_{2}(t-\tau)-\dot{x}_{1}(t)\right) \\
& \ddot{y}_{1}=-y_{1}-x_{1}^{2}+y_{1}^{2} \\
& \ddot{x}_{2}=-x_{2}-2 x_{2} y_{2}+\varepsilon\left(\dot{x}_{1}(t-\tau)-\dot{x}_{2}(t)\right) \\
& \ddot{y}_{2}=-y_{2}-x_{2}^{2}+y_{2}^{2} .
\end{aligned}
$$

As is well-known, in the uncoupled case namely for $\varepsilon=0$ the dynamics has both regular and irregular behavior largely depending on the total energy

$E_{i}$ although there is also considerable initial condition dependence [42]. The individual energies are

$$
E_{i}=\frac{\dot{x}_{i}^{2}+\dot{y}_{i}{ }^{2}}{2}+\frac{x_{i}{ }^{2}+y_{i}{ }^{2}}{2}+x_{i}^{2} y_{i}-\frac{y_{i}^{3}}{3} .
$$

Shown in Fig. 10(a) are the Poincaré maps for two different initial conditions, one leading to regular motion (outer points), while one leads to chaotic dynamics (inner points), at the same energy $E=0.13$, just below the dissociation limit, $E=1 / 6$.

When velocity coupling is introduced say for $\varepsilon=0.1$ and $\tau=2$, the system effectively becomes dissipative and the dynamics is attracted to the origin. Transient trajectories are shown in Fig. 10(b). The loss of energy has been discussed in a related context by Wang et. al [85] who showed that in the neighborhood of the fixed point, amplitude death occurs when the averaged total power is negative definite. In the case of the coupled Hénon-Heiles system the energy loss of the individual oscillators, $E_{i}$ and their difference $\left|E_{1}-E_{2}\right|$ are shown in Fig. 10(c) [84]). The question of whether other fixed points with nonzero asymptotic energies can be targeted so that the amplitude death reaches a nontrivial steady state remains open.

The above examples demonstrate that with a proper choice for the interaction, a variety of systems can show AD, thus making such steady states amenable to control.

\subsection{Routes to $A D$}

In this section we consider the mechanisms for amplitude death, namely the "routes" followed as parameters in the system are varied. Since the transition to $\mathrm{AD}$ is from oscillatory motion to a fixed point, an immediate question is whether there is a bifurcation to $\mathrm{AD}$ [86], in analogy with other standard routes that occur in nonlinear dynamical systems [87, 88, 89, 90]. 

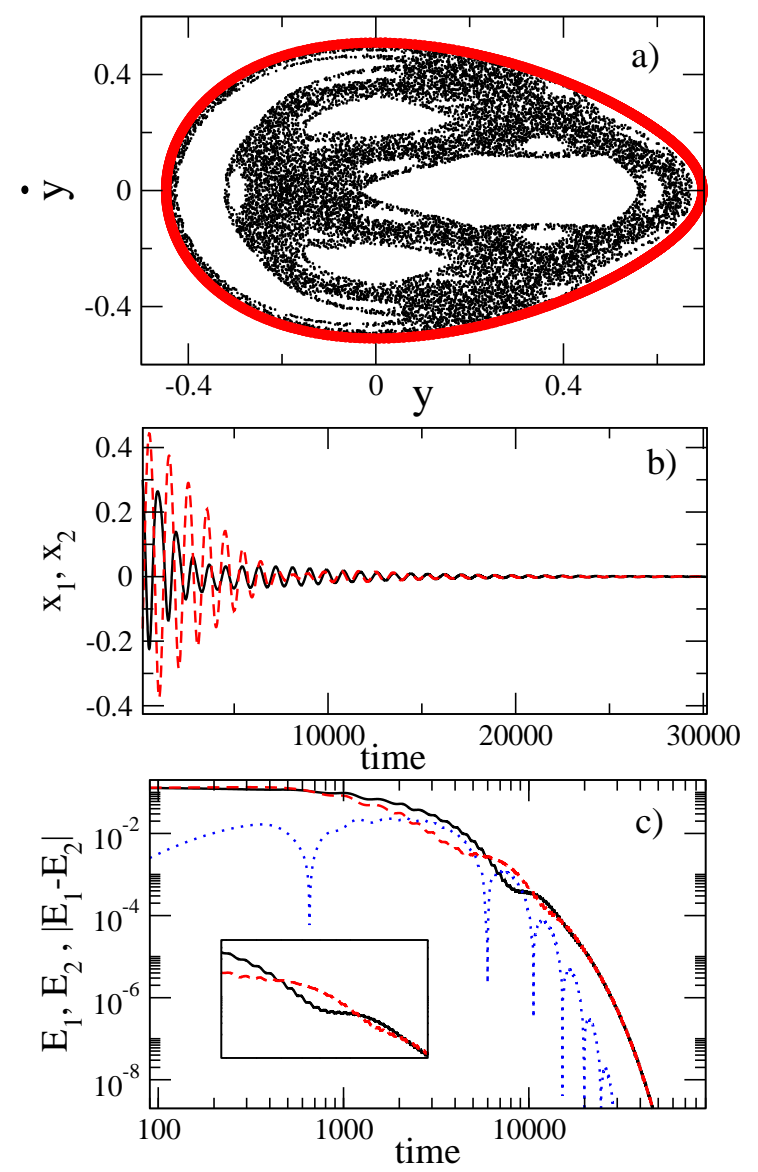

Figure 10: (Color online) (a) Poincare map at $x=0$ of uncoupled conservative HenonHeiles system, Eq. (12) at energy $E=0.13$. Outer red points and inner black points correspond to the regular and chaotic motions respectively. (b) The transient trajectories of $x_{1}$ (solid-black line) and $x_{2}$ (dashed-red line) as a function of time at coupling strength $\varepsilon=0.1$ and time-delay $\tau=2$. (c) The dissipation of energy $E_{1}$ (solid-black line), $E_{2}$ (dashed-red line) of individual oscillators and their energy difference $\left|E_{1}-E_{2}\right|$ (dottedblue line) as a function of time. Inset figure show the repeated crossing of energies while decaying (Ref. 84]). 


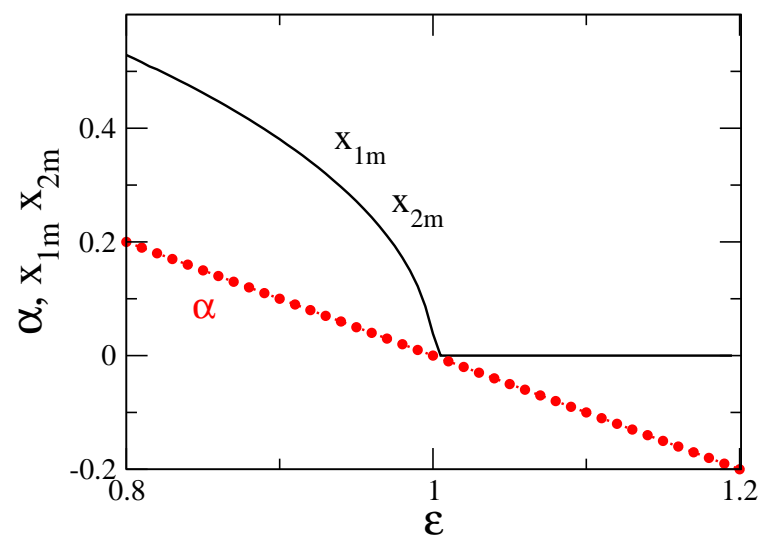

Figure 11: (Color online) Maxima of $x_{1}$ and $x_{2}$ (overlapped solid lines) and real part of the largest eigenvalues (dashed line with circles) as a function of coupling strength, $\varepsilon$; see Eq. (6).

\subsubsection{Hopf bifurcation}

The most widely observed route to AD is through the Hopf bifurcation, where the coupling induces stability of the fixed point of the uncoupled systems. In the Landau-Stuart oscillators, Eq. (66), the uncoupled system has two attractors, namely the stable limit cycle and an unstable fixed point. At the transition that occurs at $\varepsilon=1$ (see Fig. 4) the real part of the largest eigenvalue at the origin, $Z_{i}^{*}=0$ becomes negative: this is the standard Hopf bifurcation since in fact a pair of eigenvalues cross the axis from right to left in the complex plane [88]. The amplitudes also go to zero at the same point, namely $\varepsilon=1$, as shown in Fig. 11 for different frequencies, $\omega_{1}=4$ and $\omega_{2}=8$.

\subsubsection{Saddle-node Bifurcation}

Another route to AD when new fixed points are created is via a saddlenode bifurcation. These new fixed points annihilate the periodic orbit, thereby causing the oscillations to stop.

For illustration consider conjugate coupled Landau-Stuart oscillators (cf. Eq. (묘)

$$
\begin{aligned}
& \dot{x}_{1}=P_{1} x_{1}-\omega_{1} y_{1} \\
& \dot{y}_{1}=P_{1} y_{1}+\omega_{1} x_{1}+\varepsilon x_{2} .
\end{aligned}
$$

The coupling, which is nondiffusive results in the creation of new a fixed 
point [11], in addition to the origin $(0,0,0,0)$, at $\left(x_{1}^{*}, y_{1}^{*}, x_{2}^{*}, y_{2}^{*}\right)$ where

$$
\begin{aligned}
& x_{1}^{*}= \pm \sqrt{\left(\omega / \varepsilon\left(1 \pm \sqrt{\varepsilon \omega-\omega^{2}}\right.\right.} \\
& y_{1}^{*}= \pm \sqrt{1-\left(x_{1}^{*}\right)^{2} \pm \sqrt{\varepsilon \omega-\omega^{2}}},
\end{aligned}
$$

with $x_{2}^{*}=-x_{1}^{*}$ and $y_{2}^{*}=-y_{1}^{*}$. Note that this second fixed point depends on the coupling strength, and exits only when $\varepsilon>\omega$ (see the filled and open circles in Fig. 12(a)). The trivial fixed point $(0,0,0,0)$ (open diamond in Fig. 12(b)) which exists for all values of coupling strengths is always unstable, but the stability of the nontrivial fixed point changes with coupling. The stable and unstable points collide at $\varepsilon=\omega$, causing the periodic motion: thus amplitude deathcan be achieved with such a saddle-node bifurcation [86]. Note also that the oscillators settle on different fixed points that depend upon initial conditions and thus this is an instance of inhomogeneous amplitude death. This route to AD has been found in other systems as well [86].

The largest Lyapunov exponent, shown in Fig. 12(b) shows strong fluctuations in the region prior to the $\mathrm{AD}$ transition, namely the marked box $\varepsilon \in\left[\omega,\left(1+\omega^{2}\right) / \omega\right]$ suggesting that there is multistability with both periodic motion, $\left(\lambda_{1}=0\right)$ and $\mathrm{AD}\left(\lambda_{1}<0\right)$ coexisting. Details are given in [11]. This indicates that apart from the nontrivial fixed point solutions there is also a periodic solution. Multistability with a riddled basin has been seen in diffusively coupled Rössler chaotic oscillators [11] as well as dissimilar oscillators (Rössler and Lorenz systems) [26, 80]. In the riddled region, vanishingly small changes in initial conditions lead to different attractors, making the system completely unpredictable [87, 91]. This show that near the onset of AD there is the possibility of complex dynamics [11, 26, 86, 92] as well.

\subsubsection{Direct transition}

Apart from the Hopf and saddle-node bifurcations, a third type of transition to AD has been reported. Consider the system, Eq. (10) where the interaction between the subsystems is nonlinear. Shown in Fig. 13)(a) is the largest Lyapunov exponent at higher value of coupling (extension of Fig. 8): there is a jump from periodic motion $\left(\lambda_{1}=0\right)$ to $\operatorname{AD}\left(\lambda_{1}<0\right)$. This abrupt change in the dynamics could be a bifurcation but has not been analyzed completely. Similar transitions have also been observed in coupled chaotic

oscillators as for instance two Lorenz oscillators with time-delay coupling [26]

$$
\frac{d x_{1}(t)}{d t}=-\sigma\left(x_{1}-y_{1}\right)
$$



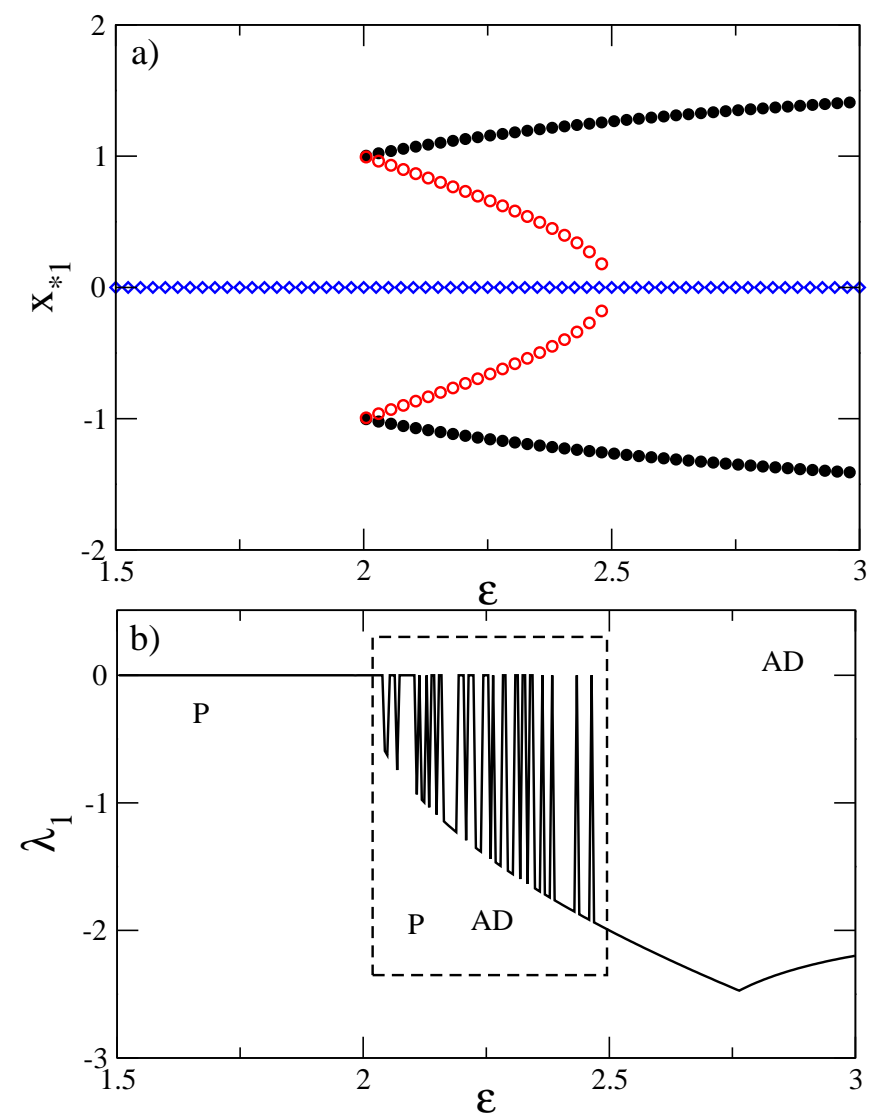

Figure 12: (Color online) (a) The $x *_{1}$ component of fixed points: trivial (blue open diamonds), nontrivial unstable (red open circles) and stable (black filled circles) with coupling strength $\varepsilon$ for conjugately one way coupled limit cycle oscillators, Eq. (14), for $\omega=2$. (b) The largest Lyapunov exponent as a function of coupling strength. Marked box shows the multistable region where periodic as well as AD co-exist [11, 86]. 


$$
\begin{aligned}
& \frac{d y_{1}(t)}{d t}=-x_{1} z_{1}-y_{1}+r_{1} x_{1}+\varepsilon\left[y_{2}(t-\tau)-y_{1}(t)\right] \\
& \frac{d z_{1}(t)}{d t}=x_{1} y_{1}-\rho z_{1} \\
& \frac{d x_{2}(t)}{d t}=-\sigma\left(x_{2}-y_{2}\right) \\
& \frac{d y_{2}(t)}{d t}=-x_{2} z_{2}-y_{2}+r_{2} x_{2}+\varepsilon\left[y_{1}(t-\tau)-y_{2}(t)\right] \\
& \frac{d z_{2}(t)}{d t}=x_{2} y_{2}-\rho z_{2} .
\end{aligned}
$$

Shown in Fig. 13(b) are a few of the largest Lyapunov exponents for $\sigma=10, r_{1}=r_{2}=28, \rho=8 / 3$, showing that all exponents become negative abruptly at a critical delay: hyperchaotic motion damps to a steady state. Such a transition is also observed in environmentally coupled systems [93], but since normal form analysis is yet to be carried out, the nature of the bifurcation remains open.

\section{Targeting and Control}

Over the last few decades the control of both periodic and chaotic oscillations in dynamical systems and the stabilization of unstable dynamics have been topics of intense research interest from both theoretical and experimental points of view [87, 94, 95, 96, 97, 98]. Several existing methods [94, 95, 96, 99, 100] stabilize fixed points by changing accessible internal pa-

rameters of a given system. In many natural systems, internal parameters are typically not accessible or at any rate cannot be tuned. Thus employing amplitude death, using appropriate interactions between the coupled systems, suggests itself as a strategy to effect control.

Very general methods are available for the design of specific fixed points in coupled nonlinear oscillators [15, 76], and some of these can be also applied to guide dynamics onto specific limit cycles. In this Section we will review studies that target fixed points which either exist in the uncoupled systems or are newly created. A related objective, the avoidance of amplitude death, the "anti-control" issue so to speak, is also discussed here.

\subsection{Steady-states through nonlinear coupling}

As discussed in Sec. 2.5, nonlinear interactions can stabilize fixed points [15, 76]. The strategy and application of this method to achieve new and 

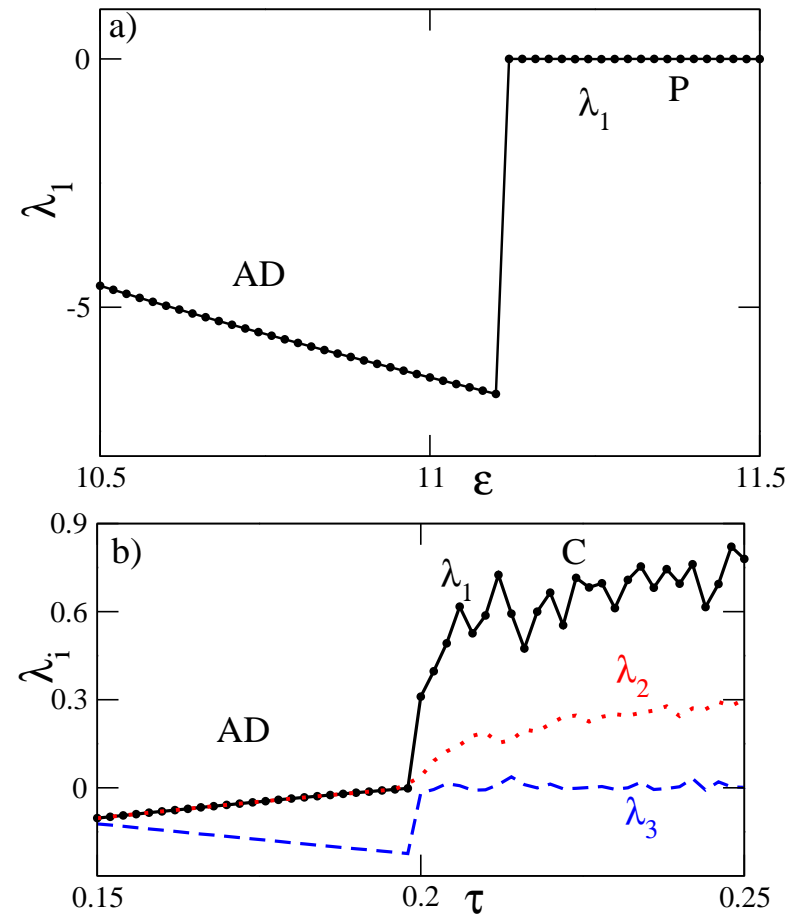

Figure 13: (Color online) The largest Lyapunov exponent as a function of coupling strength for coupled limit cycle oscillators, Eq. (6) [15], (b) The largest three Lyapunov exponents as a function of time-delay for coupled chaotic oscillators Eq. (16) at fixed coupling strength $\varepsilon=0.5[26]$. 
desirable fixed points is discussed here.

Consider coupled oscillators with a general nondiffusive coupling function $\mathbf{G}_{i}$ (see Eq. (1)). The essence of the procedure to target a fixed point is the following. Given a set of desired fixed points, $\overline{\mathbf{X}}_{i}$, these will be stationary points of the coupled system with an additional constant source, namely of the modified dynamical system

$$
d \mathbf{X}_{i} / d t=\mathbf{F}_{i}\left(\mathbf{X}_{i}\right)+\varepsilon \mathbf{G}_{i}(\mathbf{X})-\mathbf{F}_{i}\left(\overline{\mathbf{X}}_{i}\right) .
$$

The source function $\mathbf{F}_{i}\left(\overline{\mathbf{X}}_{i}\right)$ takes a constant value that depends on the desired fixed points. For suitable $\mathbf{G}_{i}$ it can be arranged that $\mathbf{G}_{i}(\overline{\mathbf{X}})=0$. Upon variation of the coupling parameters such as the coupling strength $\varepsilon$ (or by including time-delay $\tau$ in $\mathbf{G}_{i}$ ), the new fixed point can be stabilized: this, effectively, is targeted amplitude death.

As an illustration we consider coupling between identical chaotic Rössler oscillators [101] with exponential coupling $\mathbf{G}_{i} \equiv\left[\left(x_{i}-\beta\right) \exp \left(x_{j}-\delta\right), 0,0\right]^{T}$ [15] where $T$ denotes the transpose. The resulting equations for the coupled system are

$$
\begin{aligned}
& \dot{x}_{1}=-y_{1}-z_{1}-\varepsilon\left(x_{1}-\beta\right) \exp \left(x_{2}-\delta\right)+\left(\bar{y}_{1}+\bar{z}_{1}\right) \\
& \dot{y}_{1}=x_{1}+a y_{1} \\
& \dot{z}_{1}=b+z_{1}\left(x_{1}-c\right),
\end{aligned}
$$

(with corresponding equations for the other subsystem).

The parameters $\beta$ and $\delta$ in $\mathbf{G}_{i}$ are introduced such that $\mathbf{G}_{i}=0$ for $x_{i}=\beta$ and examination of the dynamical equations gives the fixed points $\bar{x}_{i}=\beta, \bar{y}_{i}=-\beta / a$ and $\bar{z}_{i}=-b /(\beta-c), i=1,2$. The stability of this fixed point can be examined as a function of $\varepsilon$ and $\beta$ [43]. Stable (S) and unstable (U) regions are indicated in Fig. 14: the unstable solution corresponds to unbounded motion, while the stable region corresponds to the possibility of the $\mathrm{AD}$ solution. For $\beta=1$, the real part of the largest eigenvalue $\operatorname{Re}(\lambda)$ [43] at the fixed point, shown in Fig. 15 indicates that amplitude death occurs when $\operatorname{Re}(\lambda)$ becomes negative. Similarly the transients shown for different values of $\beta$ for fixed $\varepsilon=0.05$ in Fig. 15(b) arrive at different fixed points $\bar{x}_{i}=\beta$. A simple extension of this idea shows how an arbitrary point in the phase-space can be stabilized [76]. 


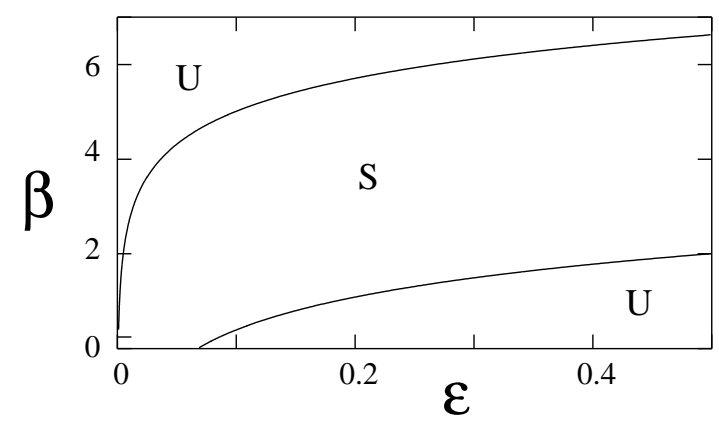

Figure 14: Schematic phase diagram in parameter space $\varepsilon-\beta$ for the coupled Rössler oscillators, Eq. (18) [76].
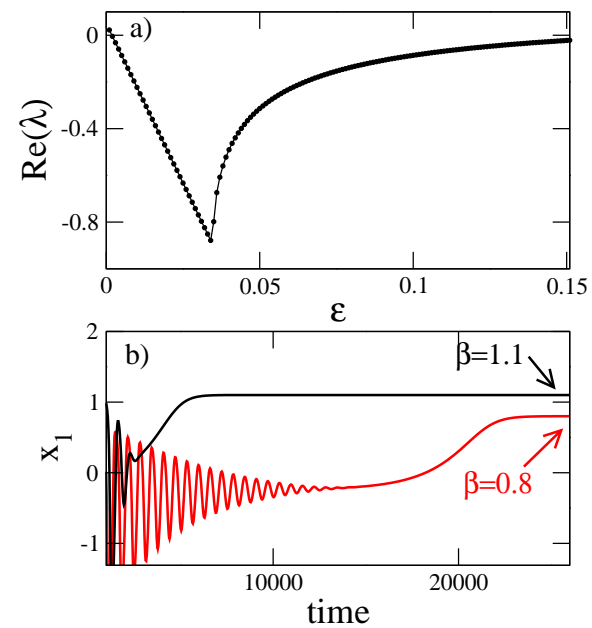

Figure 15: (Color online) (a) Real part of the largest eigenvalue (circle with solid line) as a function of the coupling strength $\varepsilon$ at $\beta=1$. (b) Transient trajectories of $x_{1}$ for $\beta=0.8$ (dashed-red line) and 1.1 (solid-black line) at coupling strength $\varepsilon=0.05$ [76]. 


\subsubsection{Linear Augmentation}

A recent scheme [77] (see Sec. 2.6) to stabilize fixed points of nonlinear systems by coupling to a linear dynamical system, $U$

$$
\begin{aligned}
\dot{X} & =F(X)+\varepsilon U, \\
\dot{U} & =-k U-\varepsilon(X-B)
\end{aligned}
$$

has some advantages. Since the $m$-dimensional linear system has the dynamics $\dot{U}=-k U$, for positive $k$, and in the absence of coupling to the nonlinear system, this is incapable of having sustained oscillations [77, 78]. The additional parameter $B$ in the augmented system thus adaptively drives the $X$ dynamics to the fixed point $B$.

This scheme is illustrated by the stabilization of arbitrary fixed points in the Lorenz oscillator 102]

$$
\begin{aligned}
\dot{x} & =\sigma(y-x)+\varepsilon u, \\
\dot{y} & =r x-y-x z, \\
\dot{z} & =x y-\rho z, \\
\dot{u} & =-k u-\varepsilon(x-b),
\end{aligned}
$$

by coupling it to a linear system as above. The fixed points when $\varepsilon=0$ are $\left(x_{0}= \pm \sqrt{\rho(r-1)}, y_{0}=x_{0}, z_{0}=r-1\right)$. For a the usual set of parameter values, $\sigma=10, r=28$, and $\rho=8 / 3$, these fixed points are unstable, and the system dynamics is chaotic.

Shown in Fig. 16] is a schematic phase diagram in the parameter space $b-\varepsilon$, the shaded regime $(\mathrm{O})$ representing oscillatory and chaotic motion, but now with an (unshaded) AD regime, corresponding to the stabilized fixed points. In order to achieve this we set $b=x_{0}=\sqrt{\rho(r-1)}=8.4853$ and took the decay constant $k=0.01$. The largest Lyapunov exponent $\lambda_{1}$ is shown as a function of the coupling strength in Fig. 17)(a) where the transition from chaotic motion to periodic motion and eventually to the desired fixed point $\left(x_{0}=y_{0}=\sqrt{\rho(r-1)}, z_{0}=r-1\right)$. A typical transient trajectory of nonlinear oscillator in the $\mathrm{AD}$ regime at $\varepsilon=6.8$ is shown in the inset.

Similar results are also observed for other fixed points; see Fig. 16 where $\mathrm{AD}$ occurs over a wider range of $b$. In the $\mathrm{AD}$ regime the coupling term in linear subsystem system vanishes since $\varepsilon(x-b) \rightarrow 0$ as $b \rightarrow x_{0}$, reducing the dynamics to $u(t)=\exp (-k t)$ namely a decay to zero. This implies that after

a transient, the coupling term in oscillatory system will also vanish hence 


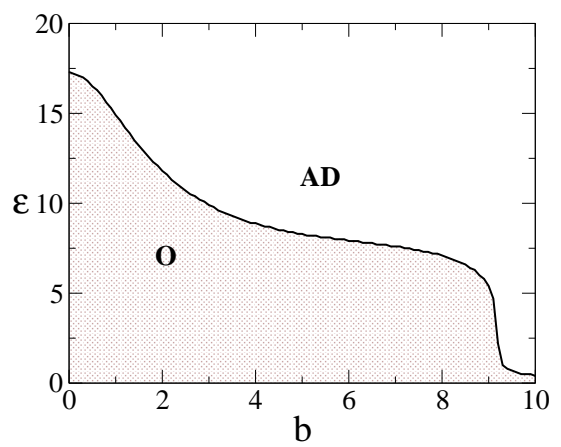

Figure 16: (Color online) Phase space diagram in parameters $(b, \varepsilon)$ for $k=0.01$ for $b=x_{0}$. Stable fixed point solution exists in the region marked $A D$, while $O$ represents the unstable steady state solution (oscillatory motion) 77 ].

stabilizing the unstable fixed point $x_{0}$ (see the inset of Fig. 17). Thus any unstable fixed point can be targeted using an appropriate value of $b$.

By choosing $b \neq x_{0}$, as the coupling terms do not vanish the system now becomes effectively 4-dimensional (in general, the augmented system has the dimension $n+m)$. In this case, there are new fixed points, and since the region of $\mathrm{AD}$ in Fig. 16 is quite wide, these can also be stabilized. Of course, this is distinct from targeting; see details in [77].

\subsection{Reprieve: Avoiding fixed points}

Some situations demand that the fixed point solution be unstable, and either chaotic or periodic solutions should be targeted. Although it is easy to perturb the system by external stimuli to avoid amplitude death, getting a specific solution is not always simple. Here we discuss methods that specifically attempt to find particular periodic solutions through targeted nonlinear interactions; see Sec. 3.1.

A modification of the coupling function $G$, for instance making it $\left\{x_{i}-\right.$ $\beta \sin (\omega t)\}$ in Eq. (18) [76] results in the dynamics being on a periodic orbit of frequency $\omega$. This behaviour is robust, namely it occurs in a specific range of parameters. If one plots the frequency of the synchronized Rössler oscillators as a function of the forcing as in Fig. 18, one can see that the common 


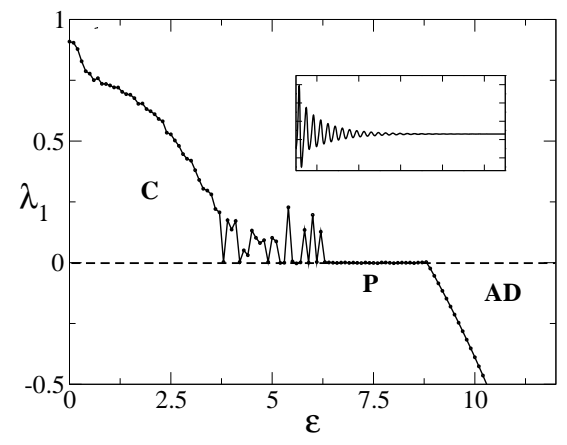

Figure 17: The largest Lyapunov exponent as a function of coupling strength, $\varepsilon$. Inset figure shows the transient trajectory, $x$, as a function of time at coupling strength $\varepsilon=6.8$, Eq. (20) [77].

frequency $\Omega$ can be made to assume any desired value. Note that the other parameters are fixed so that in the absence of periodic forcing, amplitude death would result. The input and output frequencies are identical, showing that one can indeed target periodic motion as desired. The inset figures in Figs. 18 show the time series ( $x_{1}$ vs time) associated with such a targeted periodic motion for $\omega=5$.

As indicated earlier, this method ensures only that the frequency of the targeted periodic solution takes a specific value; controlling the amplitude of the oscillations remains an open problem [76].

\subsubsection{Gradient coupling}

The gradient (or directional) coupling introduced recently [103] for $N>2$ is a new and interesting way to avoid AD. Note that amplitude death in time-delay coupled systems occurs in "islands" in parameter space (the parameters in question being the delay time $\tau$ and the coupling strength $\varepsilon$; see Sec. 2.2). In order to avoid such islands of AD asymmetry in the coupling strength, keeping the coupling function the same has been used. Consider the modification in Eq. (77) as

$$
\begin{aligned}
& \dot{Z}_{1}=\left[1+i \omega_{1}-\left|Z_{1}\right|^{2}\right] Z_{1}+(\varepsilon+r)\left[Z_{2}(t-\tau)-Z_{1}\right], \\
& \dot{Z}_{2}=\left[1+i \omega_{2}-\left|Z_{2}\right|^{2}\right] Z_{2}+(\varepsilon-r)\left[Z_{1}(t-\tau)-Z_{2}\right] .
\end{aligned}
$$

The asymmetry parameter $r$ is introduced in the coupling so that when $r=$ 0 the system is homogenous, while when $r=\varepsilon$ the coupling is anisotropic, 


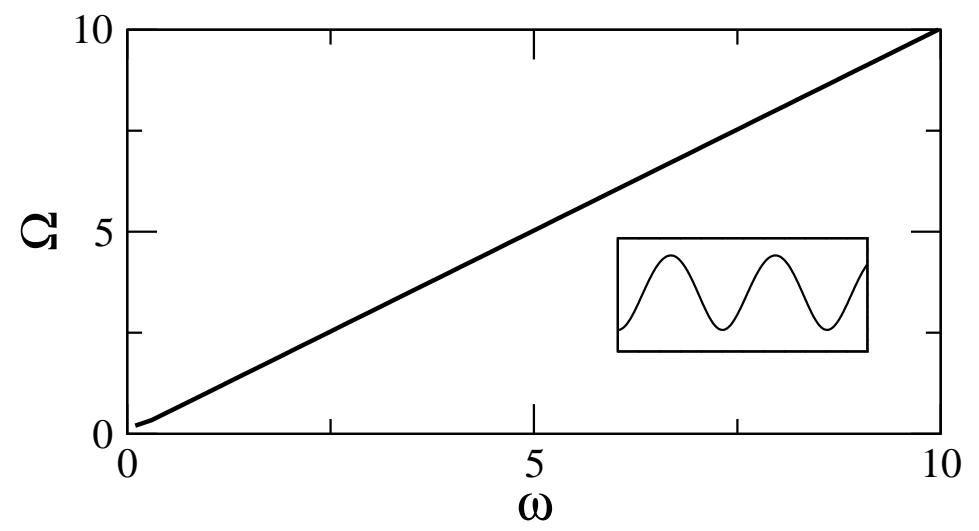

Figure 18: Variation of the common frequency of coupled Rössler oscillators $(\beta=0.4$ and $\varepsilon=0.01)$ as a function of targeted frequency $\omega[76]$.

leading to one-way coupling [103]. Such coupling arises in many practical situations [103].

Shown in Fig. 19] is a schematic phase diagram in $\tau-r$ space for fixed coupling strength $\varepsilon=2$. For $r=0$, the system behaves as shown in Fig. [5 where AD is observed within the interval $\tau \in[0.248,0.27]$. When $r$ is increased, the islands of $\mathrm{AD}$ get truncated (although the behaviour is nonmonotonic) showing that $\mathrm{AD}$ can be avoided in through interactions that have directionality. A detail analysis can be found in [103].

Konishi [104] has shown that when the Jacobian matrix at a fixed point of an isolated system has an odd number of real positive eigenvalues, then time-delay connections are not able to induce amplitude death to this fixed point. It is clear that such a property can be designed, possibly through augmentation of the system (Sec. 2.6 \& 3.1.1) into higher dimensions. This latter approach and the gradient method discussed above are of use when there is time-delay coupling. It should be emphasized that there is no general method that can be used in order to avoid amplitude death from any scenario, and thus a techniques for avoiding AD are necessary, and the problem is worthy of further study.

\section{Characterization}

Since the asymptotic dynamics in the region of amplitude death is always stationarity, the interesting dynamics are essentially the transient behaviour. Since this is also always a result of interaction between two or more systems, 


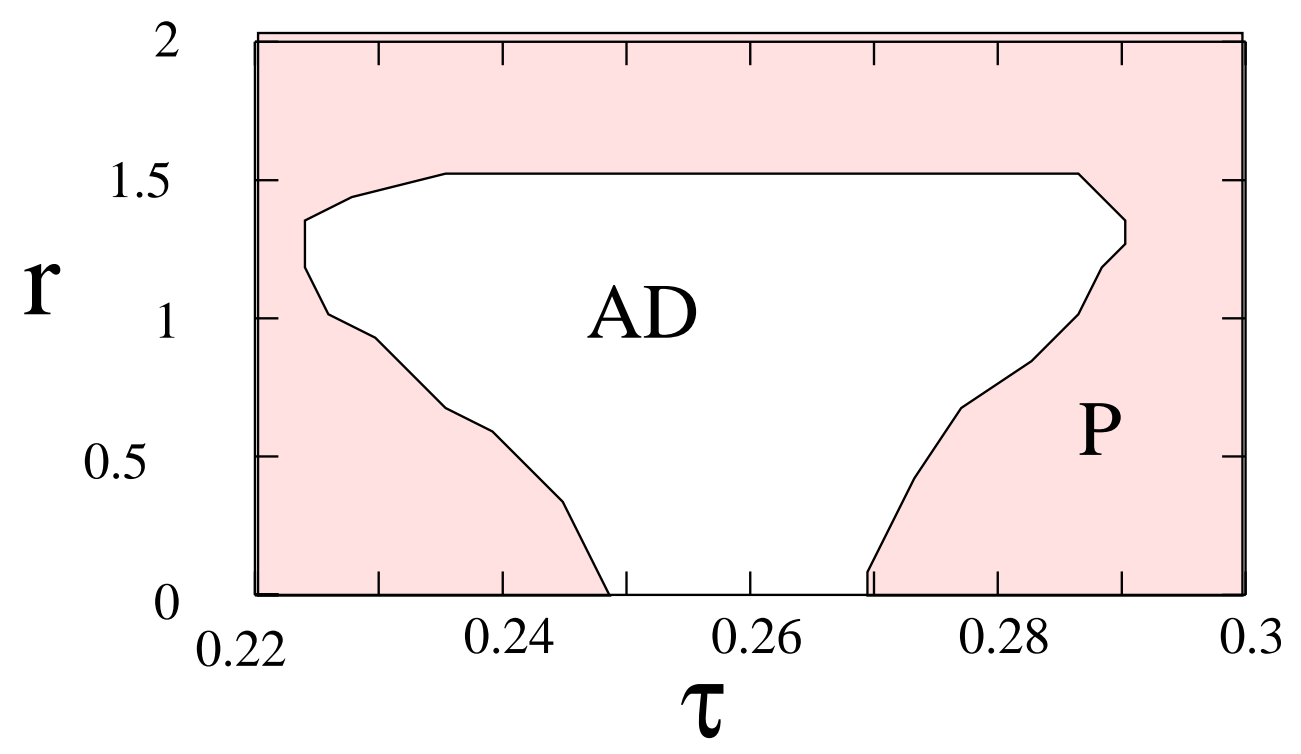

Figure 19: The schematic phase diagram in parameter space $\tau-r$ for fixed coupling strength $\varepsilon=2$, Eq. (21).

other phenomena that arise in coupled dynamics such as synchronization, riddling, and multistability can (and often do) occur, and should be taken into consideration in understanding the nature of dynamics near fixed points. In this section we focus on the nature of transient dynamics through analysis of the Jacobian matrix at the fixed point. AD can only occur when all eigenvalues have negative real parts, but the nature of the transients depend on whether there is a complex component or not.

We first consider mismatched Landau-Stuart oscillators (Sec. 2.11) without time-delay. Shown in Fig. 20(a) is the frequency of individual oscillators as a function of the coupling strength keeping other parameters as in Fig. 5. Straightforward analysis of the characteristic equation for the eigenvalues after setting $\lambda=\alpha+i \beta$ and separating real and imaginary parts gives, for the leading eigenvalue, the result

$$
\begin{aligned}
& \alpha=1-\varepsilon \\
& \beta=\left(\omega_{1}+\omega_{2}\right) / 2 \pm \sqrt{\left(\omega_{1}-\omega_{2}\right)^{2} / 4-\varepsilon^{2}}
\end{aligned}
$$

if $\varepsilon<\left(\omega_{1}-\omega_{2}\right) / 2$, while

$$
\alpha=1-\varepsilon+\sqrt{\varepsilon^{2}-\left(\omega_{1}-\omega_{2}\right)^{2} / 4}
$$




$$
\beta=\left(\omega_{1}+\omega_{2}\right) / 2
$$

when $\varepsilon>\left(\omega_{1}-\omega_{2}\right) / 2$.

This result holds when the direction of "rotation" of both oscillators is the same, which here corresponds to $\omega_{1}$ and $\omega_{2}$ having the same sign i.e. if one oscillator (in Fig. 1(a)) are rotating in clockwise then another follows the same direction. When the signs differ, the analysis is more involved, and for the case $\omega_{1}=-\omega_{2}=\omega$ (see details in Ref. [47]) the real part of the eigenvalue is unchanged (Eqs. (23) and (25)) but

$$
\beta=\sqrt{\left(\omega^{2}-\varepsilon^{2}\right)}
$$

for $\varepsilon<\omega$, while $\beta=0$ if $\varepsilon>\omega$.

Shown in the insets of Figs. 20(a) and (b) are the transients for both cases, namely co- and counter-rotating coupled oscillators. Fig. 20(c) shows the occurrence of $\mathrm{AD}$ when the signs of $\omega_{i}$ differ; compare the behaviour seen in Fig. [4 for the same extent of parameter mismatch. In Fig. 20(a) note that there is no synchrony for $\varepsilon=\left(\omega_{1}-\omega_{2}\right) / 2$, but for counter-rotating oscillators, the motion is synchronized for $\varepsilon<\omega$ while beyond this value of the coupling the system is overdamped. These results suggest that the nature of the transients thus depends on the scenarios (Sec. 21) as well as on whether the transition is from a globally synchronized state or not.

\subsection{The phase-flip}

When identical oscillators are coupled with time-delay (Sec. 2.2) they first synchronize in phase. Shown in Fig. 21(a) is the common frequency and in (b) the phase difference between the oscillators. Within the AD region, there is a point at which there is an abrupt change in the frequency and this is accompanied by a jump in phase difference by $\pi$ : the transient trajectories (inset figures) go from being synchronized in-phase to being synchronized out-of-phase behavior.

This transition, termed the phase-flip has been studied in detail in Refs. [26, 27, 28, 70, 67] in a variety of systems, as well as in different dynamical regimes. Furthermore, when there are more than two coupled oscillators, the phase-difference is not necessarily $\pi$, but can depends upon the specifics of the system as well as on the coupling topology of the network [105]. For asymmetric time-delays, even in the case of two coupled oscillators, the phase difference can be different from $\pi$ [67]. Such different phase relations have 

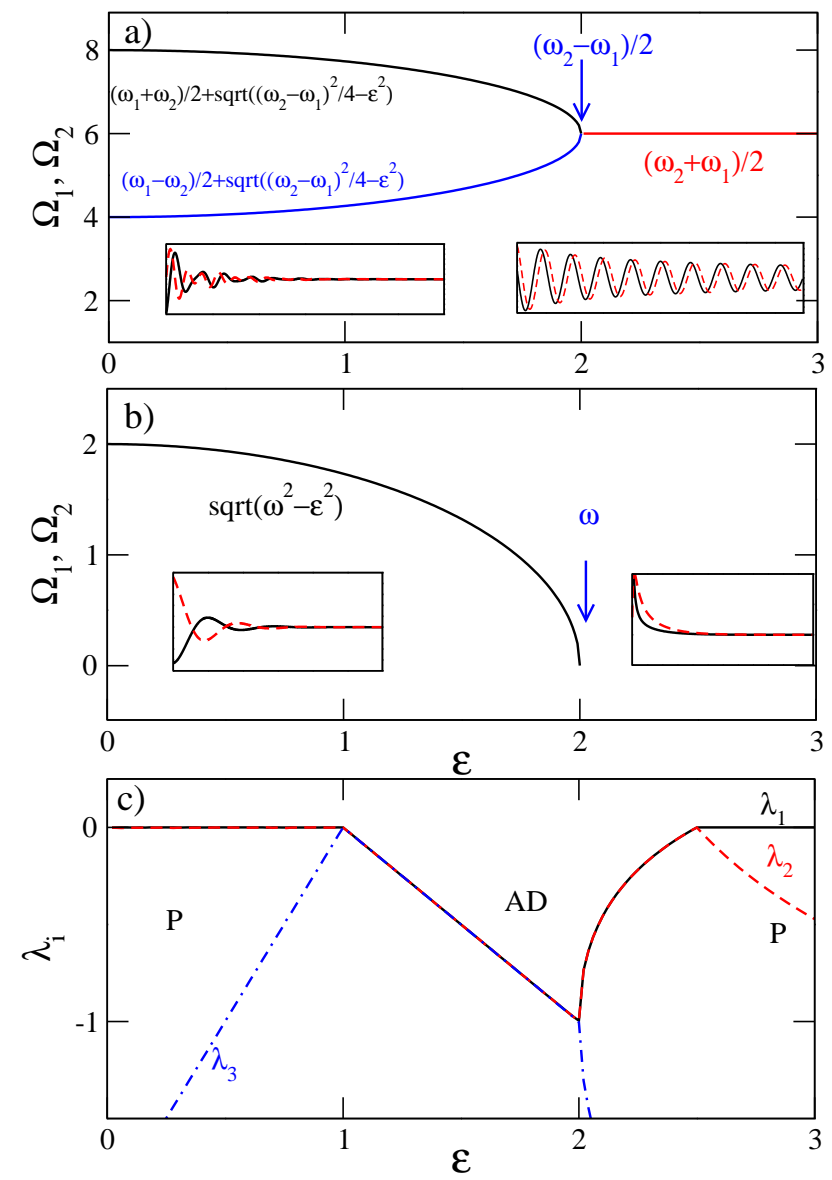

Figure 20: (Color online) Frequencies, $\Omega_{1}$ and $\Omega_{2}$, for respective oscillators for (a) mismatched, $\omega_{1}=4$ and $\omega_{2}=8$, Eq. (3) and (b) counter rotation, $\omega_{1}=2$ and $\omega_{2}=-2$ (Sec. (2.1)). (c) Largest three Lyapunov exponents for counter rotation. Inset figures show the transient trajectories in respective regimes as a function of time at coupling strengths $\varepsilon=1.5$ and $\varepsilon=2.2$. 

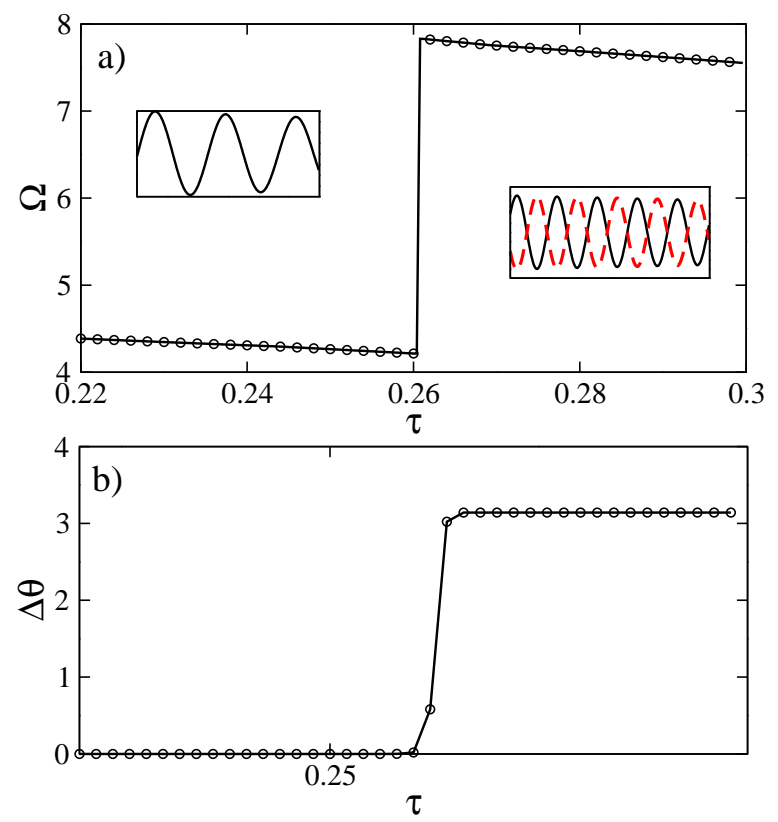

Figure 21: (Color online) (a) The common frequency of oscillations, (b) phase difference between oscillators for time-delay coupling, Eq. (3). The inset figures show the transient trajectories in respective regimes as a function of time at time-delays $\tau=0.24$ and $\tau=$ 0.28 . 
been seen in experiments with asymmetric coupling 106] and time-delay [67] as well as in in relay-coupled nonlinear oscillators [79].

As we have seen in Sec. 2.8.2 where amplitude death coexists with periodic motion, multistability can occur prior to the onset. The possibility of coexistence of both chaotic dynamics as well as stationarity has been observed in mixed Rössler and Lorenz systems [26] where there is also riddling. Although multistability is not a property of fixed point here, it should also be emphasized that at a given set of parameters reaching the fixed point solution can depend on initial conditions as well.

\section{Amplitude death on networks}

Most of the systems discussed in the previous sections are of two coupled systems only. However, many systems of interest are composed of large numbers of interacting oscillators - particularly in biological contexts - which are coupled on networks. Mathematically a network is a graph where $N$ nodes (or vertices) are connected in a specific manner. The details of the connections are an input in G (see Eq. (1)). There is considerable interest in networks in diverse areas of the sciences, spurred by the realization that these ideas have wide applicability [107]. The internet, food webs, neural networks, electrical power grids, coauthorship and citation networks of scholars, biological networks are well known instances [107, 108, 109].

The nature of the complex dynamical behavior on networks has a sensitive dependence on the nature of the connections between the nodes in addition to the actual dynamics of the individual subsystems. The occurrence of $\mathrm{AD}$ in networks of coupled oscillators [29, 30, 31, 32, 33, 34, 35] has been investigated for a variety of topologies ranging from small-world connections [38] to scale-free networks [39] and the ring topology [36, 37]. AD as an emergent phenomenon has been seen in networks of coupled maps [55] and in chaotic coupled map lattices [61].

Since one important area for the occurrence of AD is in neuronal networks, we study here a system composed of identical Hindmarsh-Rose (HR) neurons [110] that are synaptically coupled in different topologies,

$$
\begin{aligned}
\dot{x}_{i} & =a x_{i}^{2}-x_{i}^{3}-y_{i}-z_{i}-\frac{\varepsilon}{K_{i}} \sum_{j=1}^{K_{i}} A_{i j} h\left(x_{i}, x_{j}, \tau\right) \\
\dot{y}_{i} & =(a+b) x_{i}^{2}-y_{i} \\
\dot{z}_{i} & =c\left(d x_{i}+e-z_{i}\right)
\end{aligned}
$$


where

$$
h\left(x_{i}, x_{j}, \tau\right)=\frac{\left(x_{i}-V_{s}\right)}{\left[1+\exp \left\{-\beta\left(x_{j}(t-\tau)-\Theta_{s}\right)\right\}\right]} .
$$

The topology is specified through the adjacency matrix elements, $A_{i j}$, which takes the value 1 if the nodes are connected, and 0 if they are not. At each node $i$ one has a neuron, and the notation is as follows: $x_{i}$ is the membrane potential and $y_{i}$ and $z_{i}$ are the fast and slow currents respectively. The external parameters are the synaptic coupling strength $\varepsilon$ and delay $\tau$. Here the reversal potential $V_{s}$ is fixed at $V_{s}=2$ at which the synapse is excitatory, the spiking threshold is fixed at $\Theta_{s}=-0.25$ and the synaptic coupling function is taken to be sigmoidal. The other parameters are set to standard values, $a=2.8, b=1.6, c=0.001, d=9$ and $e=5$ [111].

Networks of such oscillators coupled without time-delay (namely for $\tau=$ $0)$ continue to show global amplitude death as does the case of two oscillators. Fig 22 is bifurcation diagrams for networks of $N=10$ oscillators coupled in different topologies. Three cases considered for illustration include (a) global connection, namely $K_{i}=N-1$ in Eq. (27) and all $A_{i j}=1$ for $i \neq j$, (b) a linear chain with periodic boundary conditions, $K_{i}=2$ as well as (c) random connections with $K_{i}=3$. The motion goes from being oscillatory to amplitude death as the coupling strength is increased in each of the cases, although the threshold depends on the coupling topology. In general, though, the approach to the amplitude death regime is gradual with the range of oscillation becoming narrower with increasing coupling.

The behavior of large ensembles of coupled weakly nonlinear oscillators has been studied by Ermentrout and coworkers [32] in the finite large $N$ as well as the $N \rightarrow \infty$ limits. The state of uniform rest can become stable when the coupling is sufficiently strong and the frequencies are sufficiently disparate. Amplitude death is also reported in limit cycle oscillators coupled via mean field coupling and randomly distributed frequencies [8, 9, 10, 112]. When the distribution of frequencies has sufficiently large variance, the oscillators effectively pull each other off their limit cycles into the origin which is a stable equilibrium point for the coupled systems.

A closed chain of delay coupled identical limit cycle oscillators that are near supercritical Hopf bifurcation [36] also show AD. The model is a discrete dynamical system, and a dispersion relation valid for an arbitrary number of oscillators is obtained in the continuum limit. A detailed linear stability investigation of these states has been carried out in order to delineate their 


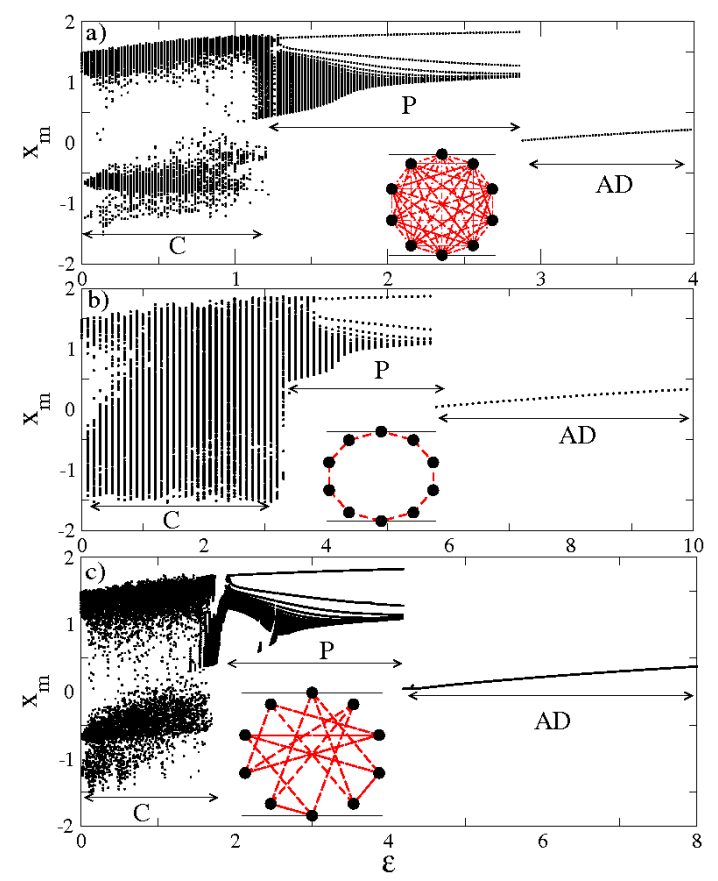

Figure 22: (Color online) Bifurcation diagrams for the system of $N=10$ coupled oscillators with (a) global coupling, (b) nearest neighbor coupling with periodic boundary conditions, and (c) a small-world network where each neuron is randomly connected to two others (shown in the insets). $x_{m}$ corresponds to the maxima of $x_{1}$ in Eq. (27) (see for details Ref. [15]). 
actual existence regions and to determine their parametric dependence on time delay. It is shown that island regions of $\mathrm{AD}$ can exist for any number of oscillators so long as the delay is finite: in effect, time delay contributes toward their stability. The size of these island is independent of $N$ when $N$ is even and is a decreasing function of $N$ when $N$ is odd. Sen and coworkers [36, 50, 52] have extensively investigated amplitude death in delay-coupled networks.

By applying averaging methods to diffusive time-delay coupled networks of weakly nonlinear oscillators [30], it is possible to determine parameter ranges in which the network experiences amplitude death [113]. Time-delay also suppresses oscillations in network of oscillators coupled in a one-way ring topology [37]. When two networks are coupled [113] delayed effects of shortcuts in each network and coupling between the two groups are considered. When the short-cut delay is removed the death of oscillations is caused by variational coupling delay. This behavior is found to hold in large number of locally coupled oscillator as well.

As already indicated, AD in neuronal networks is a phenomenon of practical interest, and there is some indication that such dynamics is significant in understanding the temporal activity appearing in the olfactory bulb [114]. In this context there has been an analytic study of neuronal models on a network with time-delayed self connection. The effect of delay on the stability of the trivial solution, and on the existence of self-sustained periodic solutions has been investigated [114]. Neural networks with distributed delay also show amplitude death [115]. When the delay distribution has dispersion below that of the exponential distribution, the system exhibits reentrance: stability is lost and then recovered when the mean delay is increased. If the delay dispersion is greater than that of the exponential distribution, the system never destabilizes.

Partial amplitude death - effectively a chimera - has been seen in a small network of globally coupled oscillators [116]. Konishi and coworkers [14, 37, 73, 74] have studied a number of models with different types of connections, on networks of varying topologies. Analytical estimation of regimes of amplitude death have been carried out, and their analysis shows that the odd-number property that is known in delayed feedback control also exits in global dynamically coupled oscillators. Furthermore global amplitude death is experimentally demonstrated in a Chua's circuit coupled by an RC line. Various transitions including amplitude death have been also investigated in a regular array of nonlinear oscillators [31]. 
A systematic procedure for achieving amplitude death involves adjusting the coupling strength and delay times in the connections [61]. Such a procedure has the advantage that stabilization can be achieved independent of the network topology. This has been applied with success to the wellknown double-scroll circuit on a small-world network (a realization can be seen in Fig. 22(c)). Hou et al. [38] investigated oscillator death behavior on small-world networks and found that small-world connectivity can eliminate the AD seen for the same model on a regular lattice. On the other hand, the small-world connectivity can also lead to global oscillator death which is absent in the regular lattice or for that matter on a completely random (Erdős-Renyi) network. On scale-free networks of nonlinear oscillators AD has been investigated both numerically and analytically in [39].

Networks of coupled scalar maps also show amplitude death as shown by Atay [117] who studied such a network with weighted connections taken so that they may include time-delay. The emergence of AD in a chaotic coupled map lattice with irregular network topologies [118] or on networks with globally dynamical interaction [73] has been investigated in recent work. Similarly, amplitude death that results from conjugate coupling has been verified theoretically in a network of chaotic oscillators under local and global conditions [68].

The Belousov-Zhabotinsky reaction in a continuous-flow stirred tank reactor (CSTR) is a well-defined nonlinear oscillator that can be realized in experiment. In addition to the study of theoretical models, experiments on networks of real systems like these also report amplitude death [119]. In the next Section we discuss several experimental systems where AD can be realized.

\section{Experiments and applications}

The stabilization of steady states, either those corresponding to the uncoupled oscillators or those created by design has been observed in various experiments. This has a variety of potential applications, and we discuss these here.

Early experimental demonstrations of AD were in coupled BelousovZhabotinskii (BZ) reactions [120], namely chemical oscillators. Other experiments on asymmetrically coupled BZ reactions [106] also lead to AD. The experimental set up used identical BZ reactors coupled via mass transfer- 
the coupling is thus diffusive in nature and since it depends on the concentration gradient both symmetric and asymmetric coupling can be realized by changing the active volumes of the tank reactors. This is of importance in biological systems where systems are indeed coupled through the exchange of fluid and are likely to be asymmetric in nature. A situation that is particularly amenable to experimental control is that of electrochemical oscillators [121]; by coupling nearly identical systems in the proximity of their individual Hopf bifurcation points, they can be driven to AD [6] as discussed in Sec. 2.1 .

Thermo-optical oscillators [122], where the coupling is linear and by heat transfer are another class of coupled systems that show AD. Thermo kinetic oscillators [123] that show temperature and concentration oscillations are of direct relevance in industrial processes. Most of these are exothermic in nature, and $\mathrm{AD}$, which has been seen experimentally in such systems can have severe consequences; these studies thus may have a significant impact on safety measures [123].

Many systems and scenarios for AD have been experimentally investigated in coupled electronic circuits. Apart from the Landau-Stuart system, the van der Pol oscillator and other standard nonlinear or chaotic oscillators that are easily realized in analog circuits, a number of hybrid models can also be studied in experiment. This extends the study of mismatched oscillators beyond the cases of dynamic coupling [37] or when the frequencies of coupled units differ [124, 125] to the situation when the coupled systems are completely distinct from each other: the interaction between an electronic and biological oscillator - a neural-electronic hybrid interface - can also suppress oscillatory dynamics [126] .

Partial amplitude death is the situation when instead of all variables reaching a stationary state, only some of them do [127]. This behaviour is seen when strong interactions break down both reflection and translational symmetry in the coupled systems, and has been experimentally verified using coupled Lorenz circuits [128]. Partial AD is distinct from the case of the chimeric behaviour in networks, where some members of a set of coupled oscillators remain oscillating while others go to a stationary state [31, 5 , 129, 130].

Experiments on coupled lasers have been a major area for the application of many ideas discussed above since delay coupling can be easily implemented. Pyragas et al. [131] have proposed that the stabilization of fluctuations in multi-mode intracavity doubled diode pumped Nd:YAG lasers can be 
achieved by stabilizing the steady state, namely through AD. A related experimental study of chaos control in lasers by Bielawski et al. [132] stabilizes the unstable steady state of a fiber two-level class B laser. Delayed coupling with delayed feedback has been shown to induce AD in a laser system [133], and Roy and coworkers [16, 17] have experimentally verified a number of issues in the dynamics of coupled systems, including amplitude death.

The fact that several meteorological phenomena are oscillatory and are frequently coupled suggests that $\mathrm{AD}$ will also occur in climate models. There are large-scale oceanic and atmospheric decadal anomalies. This variability is characterized by patterns of sea-surface temperature anomalies, correlated with the atmospheric pressure at sea level [134]. In the ocean, this variability results in fluctuations of ocean temperature and ocean mass transport. In the atmosphere these fluctuations are associated with changes in surface westerlies and surface air temperature over ocean basins and the period of these oscillations is appears to depend on the transit time of oceanic Rossby waves; these are of the order of decades. Gallego and Cessi [134], using their model for mid-latitude large scale interaction between the upper ocean and the troposphere consider the case when two ocean basins are coupled through zonally average atmosphere. Each ocean basin also interacts with the atmosphere via wind driven torques and heat fluxes at the sea surface. When uncoupled, each individual ocean basin has sustained oscillations, but with coupling, the oscillatory anomalies decay in time, and the system eventually reaches a steady state.

This vital result in the study of climate change is thus linked to amplitude death which occurs only when the long Rossby wave delays for the two ocean basins are sufficiently different. The model formulated here uses the Rossby wave delay times to control the natural frequency of oscillation in each basin, and thus the case of AD seen here can be seen as an example of mismatched interacting oscillators as discussed in Sec. 2.1.

Other large-scale systems that show AD include ecological models as for example coupled prey predator systems [135]. Amplitude death is important in epidemical models as well, e.g. in infectious disease, its important to predict whether the infection has disappeared or the pathogen persists. Steady states in the models of pathogen-immune dynamics of infectious diseases [136] are other instances of systems where the AD state is a desirable end point, and thus methods of control, discussed in Section 4 are important in the context of intervention strategies.

The issue of amplitude death has been also discussed in the field of neural 
oscillators (See Eq. (27) and corresponding Fig. 22). Amplitude death in a neural network model [137] where the interaction is "transferable", namely in a cyclic chain of oscillators with one-way coupling and with time lag. Amplitude death has also been observed in a network of Fitzhugh-Nagumo neurons [138. Diffusive coupling is not the only means of achieving amplitude death. Phase-repulsive coupling [82] also eliminate oscillations in a population of synthetic genetic clocks.

\section{Summary}

The phenomenon of amplitude death has attracted considerable attention in the past decades following the realization that this is quite general and that this has significant practical implications.

In this review we have shown that $\mathrm{AD}$ is a general outcome in coupled nonlinear oscillator systems, occurring in a wide variety of dynamical models and for different forms of coupling. There are a number of scenarios which lead to amplitude death and we have tried to use the well-studied LandauStuart limit cycle oscillator model to illustrate these. A detailed theory - in terms of bifurcation structure - is not yet available, and this constitutes an interesting and open problem.

The asymptotics of $\mathrm{AD}$ is a featureless steady state, but the transient dynamics can be of interest, with the motion of the coupled systems having nontrivial phase relations. The frequency of the damped oscillations can also show a discontinuity: this is the phase-flip transition. We have reviewed here these various dynamical characteristics in model systems.

Targeting a specific steady state is a central issue in the study of nonlinear dynamics, particularly with regard to stabilizing low-dimensional dynamics

such as fixed points or periodic orbits. We have reviewed some aspects of targeting fixed point solutions by considering specific interactions. This will be essential if one is to be able to use the phenomenon of AD in practical applications. Targeted fixed power outputs in coupled laser systems can have significant applications in laser technology e.g. laser surgery, or laser welding and fabrication. In other practical applications $\mathrm{AD}$ is to be avoided, for instance in brain function; we have also reviewed methods for the reliable avoidance of AD. There can be significant constraints, though since many natural systems have specific and constrained coupling forms - for instance the synaptic coupling between neurons, or the diffusive interaction between nonlinear chemical systems. 
Although we have considered simple systems for purposes of illustration, AD is known to occur in higher dimensional systems as well [139, 140, 141]. Time-delay makes the dynamics effectively infinite-dimensional and thus there can be $\mathrm{AD}$ in hyperchaotic systems.

External noise is unavoidable in natural systems or in experiments. AD is quite robust with regard to noise since the Lyapunov exponents of systems undergoing AD are substantially negative; the systems are deep in the region of stability. Thus AD has been observed in numerous experiments many of which have been discussed here. This aspect deserved further study, however, since it is not clear to what extent inhomogeneous steady states can be perturbed by noise (e.g. after $\varepsilon=2.5$ in Fig. 12). In addition, the study of network is currently of great importance in various areas of study and as we have seen, AD in complex systems with different topologies is of interest. The chimeric state of partial amplitude death is, in particular, an important one that needs further exploration.

Although there has been substantial work on amplitude death, several challenges remain both at fundamental as well as applications levels. Through this review we have tried to summarize the current state of understanding of the phenomenon, as well as drawn attention to several open issues, and to highlight possible applications.

\section{Acknowledgments}

GS is supported by the Council of Scientific and Industrial Research, India through a Senior Research Fellowship. AP acknowledges the support of Delhi University and the kind hospitality and financial support from the MPI-PKS Dresden, Germany. We are grateful to R. Karnatak, N. Punetha, M. D. Shrimali, S. K. Dana, and J. Kurths for useful discussions and collaboration over many years, and the Department of Science and Technology, Government of India, for research support.

\section{References}

\section{References}

[1] K. B. Eli, J. Phys. Chem., 88 (1984) 3616.

[2] A. Turing, Royal Soc. London 237 (1952) 37. 
[3] I. Prigogine and R. Lefever, J. Chem. Phys. 48 (1968) 1695.

[4] A. Koseska, E. Volkov, and J. Kurths, Europhy. Lett. 85 (2009) 28002.

[5] A. Koseska, E. Volkov, and J. Kurths, CHAOS 20 (2010) 023132.

[6] D. G. Aronson, G. B. Ermentrout, and N. Kopell, Physica D 41 (1990) 403.

[7] D. V. R. Reddy, A. Sen and G. L. Johnston, Phys. Rev. Lett. 80 (1998) 5109.

[8] P. C. Mathews and S. Strogatz, Phys. Rev. Lett. 65 (1990) 1701.

[9] R. E. Mirollo and S. Strogatz, J. Stat. Phys 60 (1990) 245.

[10] P. C. Mattews, R. E. Mirollo and S. Strogatz, Physica D 52 (1991) 293

[11] R. Karnatak, N. Punetha, A. Prasad, and R. Ramaswamy, Phys. Rev. E 82 (2010) 046219.

[12] R. Karnatak, R. Ramaswamy, and A. Prasad, Phys. Rev. E 76 (2007) 035201.

[13] F. M. Atay, Phys. Rev. Lett. 91 (2003) 094101.

[14] K. Konishi, Phys. Rev. E 68 (2003) 067202.

[15] A. Prasad, M. Dhamala, B. M. Adhikari, and R. Ramaswamy, Phys. Rev. E 81 (2010) 027201.

[16] M.-Y. Kim, Ph. D. Thesis (University of Maryland, 2005).

[17] M.-Y. Kim, R. Roy, J. L. Aron, T. W. Carr, and I. B. Schwartz, Phys. Rev. Lett. 94 (2005) 088101.

[18] P. Kumar, A. Prasad, and R. Ghosh, J. Phys. B 41 (2008) 135402.

[19] P. Kumar, A. Prasad, and R. Ghosh, J. Phys. B 42 (2009) 145401.

[20] V. P. Gangwar, A. Prasad, and R. Ghosh, J. Phys. B 44 (2011) 235403.

[21] A. Prasad, Y. C. Lai, A. Gavrielides, and V. Kovanis, Phys. Lett. A 318 (2003) 71. 
[22] J. C. Sackellares, L. D. Iasemidis, R. L. Gilmore, and S. N. Roper, in Chaos in the Brain?, Eds. K. Lehnertz, J. Arnhold, P. Grassberger, and C. E. Elger (World Scientific, Singapore, 2000), pp. 112.

[23] L. D. Iasemidis, IEEE Trans. Biom. Eng. 50 (2003) 549.

[24] A. Hastings, Ecology Letters 4 (2001) 215.

[25] A. Pikovsky, M. Rosenblum, and J. Kurths Synchronization: A Universal Concept in Nonlinear Sciences (Cambridge University Press, 2001).

[26] A. Prasad, Phys. Rev. E 42 (2005) 056204.

[27] A. Prasad, J. Kurths, S. K. Dana, and R. Ramaswamy, Phys. Rev. E 74 (2006) 035204.

[28] A. Prasad, S. K. Dana, R. Karnatak, J. Kurths, B. Blasius, and R. Ramaswamy, CHAOS 18 (2008) 023111.

[29] L. L. Rubchinsky, M. M. Sushchik, and G. V. Osipov, Math. Comput. Simulat. 58 (2002) 443.

[30] F. M. Atay, Physica D 41 (1990) 403.

[31] J. Yang, Phys. Rev. E 76 (2007) 016204.

[32] G. B. Ermentrout, Physica D 41 (1990) 219.

[33] Q.-Y. Wang, Z.-S. Duan, G.-R. Chen, and Q.-Shao Lu, Commun. Theor. Phys. 51 (2009) 684.

[34] H.-L. Chen and J.-Zhong Yang, Commun. Theor. Phys. 51 (2009) 460.

[35] X. Wang, Int. Joint. Conf. Neural Networks 3 (1992) 517.

[36] R. Dodla, A. Sen and G. L. Johnston, Phys. Rev. E 69 (2004) 056217.

[37] K. Konishi, Phys. Rev. E 70 (2004) 066201.

[38] Z. Hou and H. Xin, Phys. Rev. E 68 (2003) 055103.

[39] W. Liu, X. Wang, S. Guan, and C. H. Lai, New J. Phys. 11 (2009) 093016. 
[40] L. D. Landau, C. R. Dokl. Acad. Sci. USSR 44 (1944) 311.

[41] J. T. Stuart, J. Fluid Mech. 9 (1960) 353.

[42] M. Tabor, Chaos and Integrability in Nonlinear Dynamics: An Introduction (Wiley, John \& Sons, 1989).

[43] W. H. Press, B. P. Flannery, S. A. Teukolsky, and W. T. Vetterling, Numerical Recipes (Cambridge University Press, 2007).

[44] M. F. Crowley and R. J. Field, Nonlinear Phenomena in Chemical Dynamics (Springer-Verlag, Berlin, 1981).

[45] K. B. Eli, Phys. Chem. Chem. Phys. 13 (2011) 11606.

[46] V. R. Kumar, V. K. Jayaram, B. D. Kulkarni, and L. K. Doraiswamy, Chem. Eng. Sci. 38 (1983) 673.

[47] A. Prasad, Chaos, Solitons and Fractals 43 (2010) 42.

[48] S. K. Bhowmick, D. Ghosh, and S. K. Dana, CAHOS 21 (2011) 03311.

[49] A. Sharma and M. D. Shrimali, Nonlinear Dynamics, 69 (2012) 371.

[50] D. V. R. Reddy, A. Sen, and G. L. Johnston, Physica D 129 (1999) 15.

[51] D. V. R. Reddy, A. Sen, and G. L. Johnston, Phys. Rev. Lett. 85 (2000) 3381.

[52] D. V. R. Reddy, A. Sen and G. L. Johnston, Physica D 144 (2000) 335 .

[53] M. Lakshmanan and D.V. Senthilkumar Dynamics of Nonlinear TimeDelay Systems (Springer, Berlin, 2010).

[54] S. H. Strogatz, Nature 394 (1998) 316.

[55] F. M. Atay, Physica D 183 (2003) 1.

[56] G. Saxena, A. Prasad, and R. Ramaswamy, Phys. Rev. E 82 (2010) 017201.

[57] G. Saxena, A. Prasad, and R. Ramaswamy, Pramana-J. Phys. 77 (2011) 865. 
[58] W. Michiels, V. V. Assche, and S.-I. Niculescu, IEEE Trans. Autom. Cont. 50 (2005) 493.

[59] A. Gjurchinovski and V. Urumov, Europhys. Lett. 84 (2008) 40013.

[60] K. Konishi, H. Kokame, and N. Hara, Phys. Lett. A 347 (2010) 733.

[61] K. Konishi, H. Kokame, and N. Hara, Phys. Rev. E 81 (2010) 016201.

[62] K. Konishi and H. Kokame, in: Proc. of International Symposium on Nonlinear Theory and its Applications, 2008, pp. 528.

[63] Y. Song, J. Xu, and T. Zhang, CHAOS 21 (2011) 023111.

[64] X. Lia, J.C. Jib, and H. Hansen, Mechanics Research Communications 33 (2006) 614.

[65] W. Zou and M. Zhan, Phys. Rev. E 80 (2009) 065204.

[66] Z. Sun, W. Xu, X. Yang, and T. Fang, Chaos, Solitons and Fractals 31 (2007) 39.

[67] N. Punetha, R. Karnatak, A. Prasad, J. Kurths, and R. Ramaswamy (2012).

[68] X. Zhang, Y. Wu, and J. Peng, Int. J. Bif. Chaos 21 (2011) 225.

[69] F. Takens, in Detecting Strange Attractors in Turbulence, Lecture Notes in Mathematics, Eds. D. Rand and L. Young (Springer, New York, 1981) pp. 366 .

[70] R. Karnatak, R. Ramaswamy, and A. Prasad, CHAOS 19 (2009) 033143.

[71] M. Dasgupta, M. Rivera, and P. Parmananda, CHAOS 20 (2010) 023126.

[72] T. Singla, N. Pawar, and P. Parmananda, Phys. Rev. E 83 (2011) 026210 .

[73] K. Konishi, Int. J. Bif. Chaos 17 (2007) 2781.

[74] K. Konishi, K. Senda, and H. Kokame, Phys. Rev. E 78 (2008) 056216.

[75] K. Konishi and N. Hara, Phys. Rev. E 83 (2011) 036204. 
[76] A. Prasad, M. Dhamala, B. M. Adhikari, and R. Ramaswamy, Phys. Rev. E 82 (2010) 027201.

[77] P. R. Sharma, A. Sharma, M. D. Shrimali, and A. Prasad, Phys. Rev. E 83 (2011) 067201.

[78] V. Resmi, G. Ambika, and R. E. Amritkar, Phys. Rev. E 81 (2010) 046216 .

[79] A. Sharma, M. D. Shrimali, A. Prasad, R. Ramaswamy, and U. Feudel, Phys. Rev. E 84 (2011) 016226.

[80] Y. Chen, J. Xiao, W. Liu, L. Li, and Y. Yang, Phys. Rev. E 80 (2009) 046206 .

[81] A. N. Pisarchik, Phys. Lett. A 318 (2003) 65.

[82] E. Ullner, A. Zaikin, E. I. Volkov, and J. G. Ojalvo, Phys. Rev. Lett. 99 (2007) 148103.

[83] F. G. Lin and H. W. Ping, Chinese Phys. 16 (2007) 2825.

[84] G. Saxena, A. Prasad, and R. Ramaswamy -(unpublished).

[85] Z. Wang and H. Hu, Proceedings of International Design Engineering Technical Conferences \& Computers and Information in Engineering Conference (2005) 3.

[86] J. J. S. Vargas, J. A. Gonzalez, A. Stefanovaska, and P. V. E. McClintock, Europhys. Lett. 85 (2009) 38008.

[87] E. Ott, Chaos in Dynamical Systems (Cambridge University Press, 1993).

[88] S. H. Strogatz, Nonlinear Dynamics And Chaos: With Applications To Physics, Biology, Chemistry, And Engineering (Westview Press, 2001).

[89] Y Kuznetsov, Elements of Applied Bifurcation (Springer, New York, 2004).

[90] K. Kaneko, Theory and applications of coupled map lattices (Wiley, New York, 1993). 
[91] J. C. Alexander, J. A. Yorke, Z. You, and I. Kan, Int. J. Bif. Chaos 2 (1992) 795.

[92] A. Prasad, Y. C. Lai, A. Gavrielides, and V. Kovanis, Phys. Lett. A 314 (2003) 44.

[93] V. Resmi, G. Ambika, and R. E. Amritkar, Phys. Rev. E 84 (2011) 046212 .

[94] I. B. Schwartz, T. W. Carr, and I. Triandaf, CHAOS 7 (2997) 664.

[95] I. Triandaf and I. B. Schwartz, Phys. Rev. E 62 (2000) 3529.

[96] S. Sinha, J. S. Rao, and R. Ramaswamy, Physica D 43 (1990) 118.

[97] K. Pyragas, Phys. Rev. E 61 (2000) 3721.

[98] K. Pyragas, Phys. Lett. A 170 (1992) 421.

[99] E. Ott, C. Grebogoi, and J. A. Yorke, Phys. Rev. Lett. 64 (1990) 1196.

[100] E. Schöll and H. G. Schuster, Handbook of Chaos Control (Wiley-VCH, Weinheim 2007).

[101] E. Rössler, Phys. Lett. A 57 (1979) 397.

[102] E. N. Lorenz, J. Atmos. Sci. 20, (1963) 130.

[103] W. Zou, C. Yao, and M. Zhan, Phys. Rev. E 82 (2010) 056203.

[104] K. Konishi, Phys. Lett. A 341 (2005) 401.

[105] B. M. Adhikari, A. Prasad, and M. Dhamala, CHAOS 21 (2011) 023116.

[106] M. Yoshimoto, Chem. Phys. Lett. 280 (1997) 539.

[107] R. Albert and A.-L. Barabasi, Rev. Mod. Phys. 74 (2002) 47.

[108] S. Boccaletti, J. Kurths, G. Osipov, D.L. Valladares, and C.S. Zhou Physics Reports 1 (2002) 366.

[109] S. Boccaletti, V. Latora, Y. Moreno, M. Chavez, and D.-U. Hwang Physics Reports 175 (2006) 424. 
[110] J. L. Hindmarsh and R. M. Rose, Proc. R. Soc. Lond. B221 (1984) 87.

[111] I. Belykh, E. de Lange, and M. Hasler, Phys. Rev. Lett. 94 (2005) 188101.

[112] G. B. Ermentrout and N. Kopell, SIAM. J. Appl. Math. 50 (25) 1990.

[113] C. Y. Cheng, Phys. Lett. A. 374 (2009) 178.

[114] L. H. A. Monteiro, J. Biol. Sys. 15 (2007) 49.

[115] T. Omi and S. Shinomoto, Phys. Rev. E. 77 (2008) 046214.

[116] C. H. Ling and Y. J. Zhong, Commun. Theor. Phys. 51 (2009) 460.

[117] F. M. Atay and O. Karaback, SIAM J. Applied Dynamical Systems 5 (2006) 508.

[118] K. Konishi, Int. J. Bif. Chaos 20 (2010) 3351.

[119] M. Yoshimoto and K. Yoshikawa, Phys. Rev. E 47 (1993) 2864.

[120] K. B. Eli and S. Reuveni, J. Phys. Chem. 89 (1985) 1329.

[121] Y. Zhai, I. Z. Kiss, and J. Hudson, Phys. Rev. E 69 (2004) 026208.

[122] M. He, X. Ma, and W. Zang, Phys. Rev. Lett. 84 (2000) 5312.

[123] K. P. Zeyer, M. Mangold, and E. D. G. Peng, J. Phys. Chem. A 105 (2001) 7216.

[124] Y. Setou, Y. Nishio, and A. Ushida, IEICE Trans. Fundamentals, 79 (1996) 1575.

[125] T. Okamoto, Y. Nishio, and A. Ushida, IEICE Trans. Fundamentals 80 (1997) 1324.

[126] I. Ozden, S. Venkataramani, M. A. Long, B. W. Connors, and A. V. Nurmikko, Phys. Rev. Lett. 93 (2004) 158102.

[127] W. Liu, J. Xiao, and J. Yang, Phys. Rev. E 72 (2005) 057201.

[128] L. W. Qing, Y. J. Zhong, and X. J. Hua, Chin. Phys. 15 (2006) 2260. 
[129] K. Konishi, Proc. of IEEE International Symposium on Circuits and Systems, (Vancouver, Canada, 2004) pp-792.

[130] E. I. Volkov and D. V. Volkov, Phys. Rev. E 65 (2002) 046232.

[131] K. Pyragas, F. Lange, T. Letz, J. Parisi, and A. Kittel, Phys. Prev. E. 61 (2000) 3721.

[132] S. Bielawski, M. Bouazaoui, D. Derozier, and P. Glorieux, Phys. Rev. A 47 (1993) 3276.

[133] R. Vicente, S. Tang, J. Mulet, C. R. Mirasso, and J. M. Liu, Phys. Rev. E 73 (2006) 047201.

[134] B. Gallego and P. Cesso, J. Clim. 14 (2001) 2815.

[135] C. W. Eurich, A. Thiel, and L. Fahse, Phys. Rev. Lett. 94 (2005) 158104 .

[136] M. Neamtu, L. Buliga, F. R. Horhat, and D. Opris, Math. Mod. Nat. Phenom. 2 (2007) 44.

[137] M. He, X. Ma, and W. Zang, Discrete and Continuous Dynamical Systems, 7 (2001) 737.

[138] M. Gassel, E. Glatt, and F. Kaiser, Fluctuations and Noise Lett. 7 (2007) 225.

[139] M. Stich, A. C. Casal and J. I. Diaz, Phys. Rev. E 76 (2007) 036209.

[140] H. Sakaguchi and D. Tanaka, Phys. Rev. E 76 (2007) 025201.

[141] Z. Neufeld, I. Z. Kiss, C. Zhou, and J. Kurths, Phys. Rev. Lett. 91 (2003) 084101. 